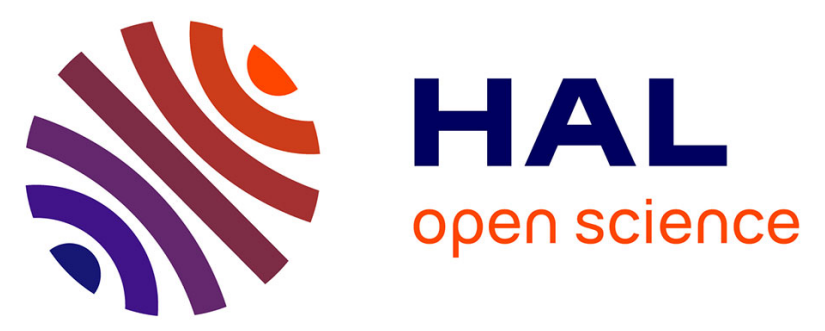

\title{
A theoretical model to analyze the Central to Eastern Pacific El Niño continuum
}

Yves Morel, Sulian Thual, Thierry Delcroix, Nick Hall, Gaël Alory

\section{To cite this version:}

Yves Morel, Sulian Thual, Thierry Delcroix, Nick Hall, Gaël Alory. A theoretical model to analyze the Central to Eastern Pacific El Niño continuum. Ocean Modelling, 2018, 130, pp.140-159. 10.1016/j.ocemod.2018.07.006 . hal-02349698

\section{HAL Id: hal-02349698 \\ https://hal.science/hal-02349698}

Submitted on 5 Nov 2019

HAL is a multi-disciplinary open access archive for the deposit and dissemination of scientific research documents, whether they are published or not. The documents may come from teaching and research institutions in France or abroad, or from public or private research centers.
L'archive ouverte pluridisciplinaire HAL, est destinée au dépôt et à la diffusion de documents scientifiques de niveau recherche, publiés ou non, émanant des établissements d'enseignement et de recherche français ou étrangers, des laboratoires publics ou privés. 


\title{
A theoretical model to analyze the Central to Eastern Pacific El Niño continuum
}

\author{
Yves Morel $^{1, *}$, Sulian Thual ${ }^{2, *}$, Thierry Delcroix ${ }^{1, *}$, Nick Hall $^{1, *}$, Gaël \\ Alory $^{1, *}$
}

\begin{abstract}
A current scientific issue of great interest is to understand the mechanisms leading to the localization of El Niño events either in the Central (CP) or Eastern Pacific (EP). For this, we derive a reduced gravity mixed layer model for the equatorial ocean with simple nonlinearities, diabatic effects and zonally varying background characteristics. Using the model, we study the propagation of an equatorial Kelvin wave from an initial perturbation. An approximate analytical solution is found for the evolution of the maximum density (or temperature) anomaly created during the passage of the wave. Density anomalies can either peak in the $\mathrm{CP}$ or continuously increase until reaching the EP, which is representative of both types of El Niño. Sensitivity tests reveal that both the zonally varying background stratification and diabatic effects are important to determine the density pattern. The EP pattern is obtained for smooth background variations while the CP pattern requires a frontal background structure. Using numerical experiments, we then show how consecutive Kelvin waves can lead to the transition from a $\mathrm{CP}$ to an EP pattern. The present theoretical results provide useful insights for understanding El Niño dynamics and diversity in more complete models and observations.

Keywords: Equatorial Kelvin waves, mixed layer, El Niño.
\end{abstract}

\footnotetext{
* Corresponding authors

Preprint submitted to Qcean Modelling November 5, 2019 Sulian.Thual@gmail.com (Sulian Thual), Thierry.Delcroix@legos.obs-mip.fr (Thierry Delcroix), Nick.Hall@legos.obs-mip.fr (Nick Hall), gael.alory@legos.obs-mip.fr (Gaël Alory)

${ }^{1}$ LEGOS, University of Toulouse, CNES, CNRS, IRD, UPS, Toulouse, France

${ }^{2}$ Center for Atmosphere and Ocean Science, Courant Institute of Mathematical Sciences, New York University, New York, USA
} 


\section{Introduction}

\subsection{El Niño}

The El Niño-Southern Oscillation (ENSO) is one of the most studied phenomena in oceanic and atmospheric sciences, owing to its major consewide teleconnections. The ENSO consists of alternating periods of anomalously warm El Niño conditions and cold La Niña conditions every 2 to 7 years, with considerable irregularity in strength, duration and structure of these events (Neelin et al., 1998; Djikstra, 2006; Clarke, 2008). One of the striking consequence of this phenomenon is the occurence and westward propagation of a sea surface temperature (SST) anomaly, which has major consequences for the tropical Pacific climate and fisheries. Many studies have been carried out but the details of the mechanisms setting up an El Niño event and driving its diversity and possible evolution still need to be better understood to be successfully modeled and predicted (Wang and Picaut, 2004; Ashok and Yamagata, 2009; Guilyardi et al., 2009; Cai et al., 2015; Capotondi et al., 2015).

1.2. $E P$ vs $C P$

The mechanisms and prediction of El Niño events remain elusive despite their important impacts because each of them shows unique and distinctive features. In fact, El Niño events vary greatly in strength, evolution and localization in the recent observational record. A current scientific issue of great interest is to understand the mechanisms leading to the localization of El Niño sea surface temperature (SST) anomalies either in the Central 
49 (CP) or Eastern Pacific (EP). Observations indicate two different fates for

50 El Niño events: the SST anomaly propagates up to the eastern Pacific (EP)

51 or stalls in the central Pacific (CP). While extreme El Niño events such 52 as those of 1982/83, 1997/98 have maximal SST anomalies in the eastern 53 Pacific, there have been many examples of moderates $\mathrm{CP}$ events in the 54 recent record (Ashok et al., 2007; Kug et al., 2009; Capotondi et al., 2015).

55 The recent extreme El Niño event of 2015/2016 showed SST anomalies in

56 both the central and eastern Pacific (Paek et al., 2016; Chen et al., 2017a).

57 Observational studies also show that there has been a constant increase in

58 the occurence of CP El Niño events during the last centuries and decades

59 (Liu et al., 2017), and modeling studies suggest that such a trend would

60 continue in an anthropogenic warming climate scenario (Yeh et al., 2009;

61 Cai et al., 2014).

62

1.3. modeling of $E P$ vs $C P$

63 ENSO diversity presents a major challenge to Coupled General Circula64 tion Models (CGCMs, see Clarke et al., 2007). In fact, most of those CGCMs

65 still show deficiencies in simulating the diversity of El Niño amplitude, lo-

66 calization and frequency due to systematic biases in the mean climate and

67 seasonal cycle of the tropical Pacific (Bellenger et al., 2014). However, there

68 have been examples of GCM improvements for simulating both the CP and

69 EP El Niños (Kug et al., 2010; Dewitte et al., 2012). CP and EP El Niños are

70 also captured in several simpler models based on different recipes. The ear-

71 liest models of both conceptual and intermediate complexity usually focused

72 on the dynamics of EP El Niño events only (Zebiak and Cane, 1987; Suarez

73 and Schopf, 1988; Jin, 1997). More recent theoretical studies suggest that

74 the dynamics of the CP and EP events involve different processes. While 
75 studies usually agree that the EP El Niño is driven by thermocline feedback

76 in the eastern Pacific (An and Jin, 2001; Dewitte et al., 2013), several mech-

77 anisms have been proposed for the dynamics of the CP El Niño. Simple

78 models depict the CP El Niño as resulting, for example, from the zonal ad-

79 vection of the warm pool SST edge (Picaut et al., 1997; Clarke et al., 2007)

80 or the nonlinear advection of SST anomalies in the central Pacific (Chen

81 et al., 2017b). Fedorov et al. (2015) and Chen et al. (2015) suggest that the

82 different EP and CP flavors may result from different responses to state-

83 dependent westerly wind bursts. In contrast, Ren and Jin (2013) suggest

84 that the CP and EP El Niños can be represented as two independent modes

85 of the simple recharge-discharge model from (Jin, 1997).

86 1.4. EP / CP continuum

87 The above bimodal separation of EP and CP events has been questioned 88 and recent consensus is that they are part of a continuum. For instance, 89 Capotondi et al. (2015) analyse the diversity of El Niño events in nature, 90 pointing to the $\mathrm{CP} / \mathrm{EP}$ continuum that is evidenced by the overlapping 91 localization of SSTs from the central to eastern Pacific. Takahashi et al. 92 (2011) analyses empirical orthogonal functions of SSTs suggesting that the 93 $\mathrm{CP}$ and EP El Niño regimes are non-separable and interact nonlinearly.

94 1.5. Coupled instability studies

95 Insight on ENSO dynamics has also been gained by analyzing the prop96 agation of coupled (ocean and atmosphere) equatorial waves using linear 97 instability methods. These studies focus on the coupling between the ocean 98 mixed layer(s) and atmosphere to explain the growth of the SST anomaly 99 (Hirst, 1986; Pontaud and Thual, 1998; Thual et al., 2012). While the 
above studies consider homogeneous background conditions (i.e. constant with space) for simplicity, several studies have also analyzed the effect of varying background characteristics on coupled instabilities. For this, numerical experiments are usually considered to solve the nonlinear dynamics while simplified analytical results may be obtained in some instances using common approximations such as the method of multiple scales (e.g. WKB approximation, Busalacchi and Cane, 1988; Yang and Yu, 1992). One of the most important background characteristics that affects equatorial wave propagation appears to be zonal changes in ocean stratification (e.g. in thermocline depth), which are quite marked in the equatorial Pacific. For example, a shoaling thermocline can significantly modify the characteristics of an equatorial Kelvin wave including its amplitude, deformation radius and baroclinic structure in addition to reflecting a significant portion of the flux westward (Long and Chang., 1990; Fedorov and Melville, 2000). Observational studies further suggest that this type of nonlinear equatorial wave dynamics is at work to some extent in nature (e.g. Zheng et al., 1998; Cravatte et al., 2003; Bosc and Delcroix, 2008). Such an analysis can be extended to study coupled ocean-atmosphere basin modes in the presence of boundary reflections at the eastern and western edges of the equatorial Pacific (Wakata and Sarachik, 1991; Yang and O'Brien, 1993; Wu and Anderson, 1995). While the above studies detail the propagation mechanisms of equatorial waves, they do not necessarily relate them to the mechanisms of the EP and CP continuum of El Niño events.

\subsection{Article scope and structure}

An important point is thus that very few simple process-oriented studies address the theoretical aspects of the CP El Niño or the continuity between 
CP and EP El Niño events (Capotondi et al., 2015). This is what we propose in the present study. Our aim is not to reproduce the complete evolution of El Niño events. We restrict our attention to the propagation of an equatorial Kelvin wave, once it has been formed, and analyze its effect on the modification of the mixed layer temperature, here associated with density.

To do so, we derive a reduced gravity mixed layer model for the equatorial ocean with simple nonlinearities, diabatic effects and zonally varying background characteristics (section 2). We derive an analytical expression for zonal shape of the wave and the density anomaly it generates under adiabatic (section 3) or diabatic (section 4) evolution. We analyze under which circumstances density anomalies can either peak in the $\mathrm{CP}$ or continuously increase until reaching the EP. The sensitivity to several parameters is also studied (section 5). Finally, using numerical experiments, we show how consecutive Kelvin waves can lead to the transition from a CP to an EP pattern (section 6).

\section{The model}

\subsection{Simplified equations : a bulk mixed layer $1 D$ model}

We here focus on the evolution of an equatorial Kelvin wave in a variable background state. We simplify the equations as much as possible to only retain some aspects of the dynamics: pressure gradient, horizontal advection, divergence of the horizontal velocity field and the stretching effect associated with the vertical motion of the base of the thermocline $\left(w(z=-H)=\frac{d H}{d t}\right)$. These are the main processes responsible for the wave propagation and for the impact of the wave on tracers such as density. The most simple model, able to reproduce the main features of equatorial Kelvin waves, is a bulk 
mixed layer reduced gravity model along the equator. The model is thus $1 \mathrm{D}$ in the longitude direction, the meridional velocity $V=0$ and the Coriolis term are neglected (see Fig. 1). The momentum conservation, continuity and buoyancy evolution equations then read (see Gill, 1982; Anderson and McCreary, 1985; Benestad, 1997; Neelin et al., 1998; Dijkstra and Burgers, 2002, and Appendix A for further details and justification of the model):

$$
\begin{gathered}
\partial_{t} U+U . \partial_{x} U=-\frac{\partial_{x} P}{\rho_{r e f}}+F^{U}, \\
\partial_{t} H+\partial_{x}(H U)=F^{H}, \\
\partial_{t}\left(\rho_{s} H\right)+\partial_{x}\left(\rho_{s} H U\right)=F^{\Theta},
\end{gathered}
$$

where $U$ is the longitudinal velocity field, $P$ is the hydrostatic pressure, $\rho_{\text {ref }}$ is a constant reference density such that the total density in the mixed layer is $\rho_{s}^{t o t}=\rho_{\text {ref }}+\rho_{s}$, where $\rho_{s}$ is the zonal variation of the mixed layer density, and $H$ is the mixed layer thickness. $F^{U}, F^{H}$ and $F^{\Theta}$ are forcing terms representing all effects necessary to explain the evolution of averaged physical quantities in the mixed layer, including all forcing effects (wind stress, vertical mixing, buoyancy/heat flux, effect of instabilities).

The dynamics is intensified in the upper layer and the layers below are considered at rest, so that the pressure anomaly $P$ can be expressed as a function of the mixed layer thickness and density:

$$
P \simeq g\left(\rho_{b}-\rho_{s}^{t o t}\right) H,
$$

${ }_{67}$ where $g=9.81 \mathrm{~m} . \mathrm{s}^{-2}$ is the earth gravity and $\rho_{b}$ the density of the lower layer (at rest). If we further consider that the lower layer density variation 


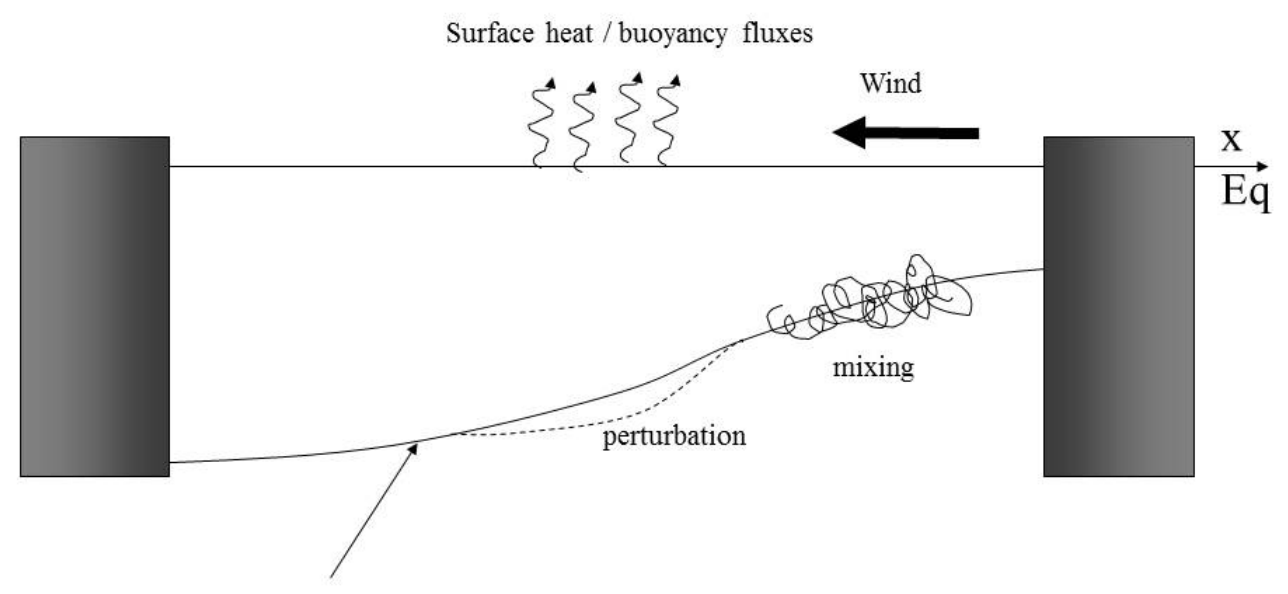

Isotherm / isopycnal surface limiting the upper layer

Figure 1: 1D (along Equator) configuration considered in the study. 
$\rho_{b}$ is negligible, we can set $\rho_{\text {ref }}=\rho_{b}$ and Eq. 1 become:

$$
\begin{aligned}
\partial_{t} U+U . \partial_{x} U & =\partial_{x}(\Theta)+F^{U}, \\
\partial_{t} H+\partial_{x}(H U) & =F^{H}, \\
\partial_{t} \Theta+\partial_{x}(\Theta U) & =F^{\Theta},
\end{aligned}
$$

170 where $\Theta=g \rho_{s} H / \rho_{\text {ref }}$ measures the local buoyancy ( $\approx$ heat) content of the 171 upper layer. Note that, as $\rho_{b}$ is taken as the reference density $\rho_{s}$ and $\Theta$ are 172 negative. Tables D.2 and D.3 in Appendix D summarize all variables and 173 parameters used in the present study.

174

As mentioned above, Eq. 3 are very simplified but retain the basic dynamics of Kelvin wave evolution. Other processes that have been proven to be important for ENSO (ocean/atmosphere fluxes, mixing at the base of the mixed layer, effects of vertical variations within the upper layer, ..., see Chen et al., 2016, for instance) will be parameterized through the forcing terms $F$ (see below).

\subsection{Configuration}

We hypothesize that there exists a known mean steady (e.g. slowly varying with respect to the wave propagation) state, $U_{o}, H_{o}, \Theta_{o}=g \rho_{o} H_{o} / \rho_{r e f}$, maintained by forcings $F_{o}^{U}, F_{o}^{H}$ and $F_{o}^{\Theta}$. The forcing terms can be calculated from the mean state using Eq. 3:

$$
\begin{aligned}
& F_{o}^{U}=U_{o} . \partial_{x} U_{o}-\partial_{x}\left(\Theta_{o}\right) \\
& F_{o}^{H}=\partial_{x}\left(H_{o} U_{o}\right) \\
& F_{o}^{\Theta}=\partial_{x}\left(\Theta_{o} U_{o}\right)
\end{aligned}
$$

5 Note that the basic state forcing terms $F_{o}$ should comprise all processes maintaining the mean state: mean forcing terms and the mean contribution 
of mixing or fluxes due to quadratic transient interactions. The Kelvin wave solutions we obtain below are perturbations of our imposed basic state and we consider them separately from any background transient activity maintaining the basic state.

We analyze the evolution of perturbations $u, h, \theta$ around the mean state and, with the decomposition $U=U_{o}+u, H=H_{o}+h, \Theta=\Theta_{o}+\theta$, the linearized equations for the perturbations are:

$$
\begin{aligned}
& \partial_{t} u+\partial_{x}\left(U_{o} u\right)=\partial_{x}(\theta)+\delta F^{U}, \\
& \partial_{t} h+\partial_{x}\left(H_{o} u+h U_{o}\right)=\delta F^{H}, \\
& \partial_{t} \theta+\partial_{x}\left(\Theta_{o} u+\theta U_{o}\right)=\delta F^{\Theta},
\end{aligned}
$$

with $\delta F^{X}=F^{X}-F_{o}^{X}$. Hereafter, $\delta F^{X}$, if not neglected, will be parameterized as a Newtonian cooling (see below). We will propose approximate analytical solutions of Eq. 5 and we will compare them to numerical solutions of the full nonlinear equations 3 .

\subsection{Density equation}

The surface density perturbation evolution equation can be re-derived from Eq. 3 and using $\rho_{s}=\rho_{r e f} \Theta / g H$ :

$$
\partial_{t} \rho_{s}+U \partial_{x}\left(\rho_{s}\right)=\frac{\rho_{r e f}}{g H} F^{\Theta}-\frac{\rho_{s}}{H} F^{H} .
$$

1 For gravity waves, $U, H$ and $\Theta$ exhibit a propagating signal at first order, but density does not exhibit the same evolution: it is a tracer and it evolves under the influence of advection and diabatic forcing. As a result, for the numerical model with the full nonlinear Eq. 3, density can be diagnosed from the numerical $\Theta$ and $H$. This is not the case for the analytical calculations, which yield approximate, linear -propagating- solutions for $U, H, \Theta$. The 
analytical calculation of density requires us to solve Eq. 6 forced by the linear solution of Eq. 5 (see below and Appendix C). We also set $\rho_{s}=\rho_{o}+\rho$ where $\rho$ is the density perturbation generated by the wave (see tables D.2 in Appendix D for a definition of all variables).

\section{Results for linear waves in adiabatic conditions}

\subsection{Analytical solutions in adiabatic conditions}

We present here analytical solutions for the linearized Eq. 5 in the case of homogenous or zonally varying background equilibrium state under adiabatic conditions (i.e. no forcing terms, $\delta F^{X}=0$ ).

First, if the background equilibrium state is homogeneous $\left(U_{o}, \Theta_{o}, H_{o}\right.$ do not vary) and we neglect forcing terms, Eq. 5 reverts to the well known gravity wave model, whose solution is:

$$
\begin{aligned}
u & =-g \rho_{o} /\left(\rho_{r e f} C_{o}\right)\left[h^{+}\left(x-\left(U_{o}+C_{o}\right) t\right)-h^{-}\left(x-\left(U_{o}-C_{o}\right) t\right)\right], \\
h & =\left[h^{+}\left(x-\left(U_{o}+C_{o}\right) t\right)+h^{-}\left(x-\left(U_{o}-C_{o}\right) t\right)\right], \\
\theta & =g \rho_{o} / \rho_{\text {ref }}\left[h^{+}\left(x-\left(U_{o}+C_{o}\right) t\right)+h^{-}\left(x-\left(U_{o}-C_{o}\right) t\right)\right],
\end{aligned}
$$

Here $C_{o}=\sqrt{-\Theta_{o}}$ is the gravity wave propagation speed, $h^{+}$and $h^{-}$are two functions defined from the initial conditions and corresponding to the eastward and westward propagation of the initial perturbation. As stated above, in the following we will consider eastward propagating waves. Note the buoyancy anomaly $\theta$ is proportional to the volume anomaly $h$ and the wave is non-dispersive. The density anomaly is null. These are well known results for gravity waves.

Secondly, when the background state varies, the previous results are no longer valid and the equations are much more complicated. Giese and Harrison (1990) have addressed this problem and have proposed expressions 
where

$$
\frac{x}{C(x)}=\int_{x_{o}}^{x} \frac{d x}{C_{o}(x)},
$$

Here again $C_{o}=\sqrt{-\Theta_{o}}$ is the gravity wave propagation speed, but now it varies zonally. $\theta_{o}$ is determined by the initial perturbation, and $x_{o}$ is the initial position of the perturbation. As shown by Eq. 8, the amplitude of the perturbations evolves during the propagation: $\theta \propto C_{o}^{-1 / 2}, h \propto \rho_{o}^{-1} C_{o}^{-1 / 2}$ and $u \propto C_{o}^{-3 / 2}$. Note that if $C_{o}(x)$ decreases during the eastward wave propagation, the amplitude of the perturbation increases, and the velocity amplitude increases faster than the buoyancy or height anomalies.

The previous solutions Eq. 8 are similar to the solutions obtained by Giese and Harrison (1990) in a different framework (see also (Benestad, 1997)).

\subsection{Analytical solutions for density evolution in adiabatic conditions}

To calculate the density perturbation created by the passage of the wave, we have to solve Eq. 6. For the adiabatic linear configuration considered in 
this section, an approximation of this equation is:

$$
\partial_{t} \rho=-u \partial_{x} \rho_{o}
$$

250

Using the solution for $u$ given by Eq. 8, an approximate solution can be derived for the maximum density perturbation (see Appendix C), and we find that the structure of the maximum density anomaly created by the passage of the wave is:

$$
\rho_{\max } \propto-\frac{\rho_{o}^{\prime}}{C_{o}^{3 / 2}}=-\frac{\rho_{o}^{\prime}}{\left(-g \rho_{o} H_{o} / \rho_{\text {ref }}\right)^{3 / 4}} .
$$

\subsection{Experimental setup in adiabatic conditions}

We now analyze the propagation of an equatorial Kelvin wave in a setup representative of the initiation of El Nino events in the equatorial Pacific. We will compare and analyze results of the full nonlinear model from Eq.36 or consider the analytical solutions from Eq. 8-11. We consider two experiments that differ only by their equilibrium states, leading to either a CP or EP pattern of density anomalies.

First, for the EP experiment the equilibrium state is specified as:

$$
\begin{aligned}
& U_{o} \simeq 0, \\
& H_{o}=H_{\text {mean }}-\Delta H_{\max } \frac{x-L / 2}{L}, \\
& \rho_{o}=\rho_{\text {mean }}+\Delta \rho_{\text {max }}^{\text {lin }} \frac{x-L / 2}{L},
\end{aligned}
$$

where $L$ is the basin width, $H_{\text {mean }}$ is the mean mixed layer depth (reached in the middle of the domain) and $\Delta H_{\max }$ its variation between the western and eastern sides, $\rho_{\text {mean }}$ is the mean mixed layer density and $\Delta \rho_{\text {max }}$ its variation between the western and eastern sides. We set $L=30.000 \mathrm{~km}$, $H_{\text {mean }}=120 \mathrm{~m}, \Delta H_{\text {max }}=160 \mathrm{~m}, \rho_{b}=0, \rho_{\text {ref }}=1000 \mathrm{~kg} / \mathrm{m}^{3}, \rho_{\text {mean }}=$ 
$267-3.0 \mathrm{~kg} / \mathrm{m}^{3}, \Delta \rho_{\max }^{\text {lin }}=3 \mathrm{~kg} / \mathrm{m}^{3}$. A large basin scale $L=30.000 \mathrm{~km}$ has ${ }_{268}$ been chosen so as to get rid of boundary effects at the western and eastern

$$
\begin{aligned}
& h(t=0)=h_{o}=\delta h_{\text {max }} \exp \left(-\left(\left(x-x_{o}\right) / l_{p}\right)^{2}\right), \\
& u(t=0)=u_{o}=\frac{\sqrt{-\Theta_{o}}}{H_{o}} h_{o}, \\
& \theta(t=0) \quad=\theta_{o}=\frac{\Theta_{o}}{H_{o}} h_{o},
\end{aligned}
$$

286 where $l_{p}$ is the perturbation lengthscale, $x_{o}$ is the initial position of the perturbation and $\delta h_{\max }$ the amplitude of the mixed layer depth anomaly. The 


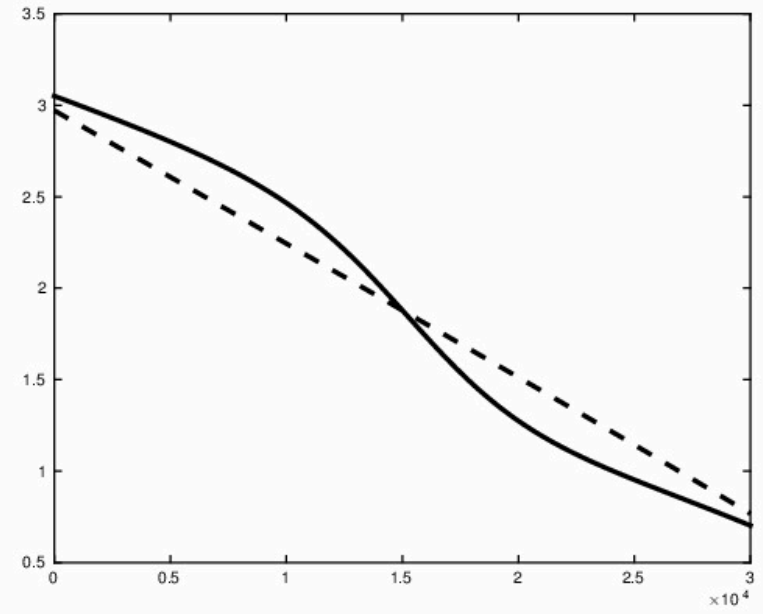

Figure 2: Gravity wave propagation speed $C_{o}$ (in $\mathrm{m} / \mathrm{s}$ ) for the EP (dashed line) and CP (solid line) experiments as a function of the zonal coordinate $x$ (in $\mathrm{km}$ ). Note the variation in the middle of the basin for the $\mathrm{CP}$. 
velocity anomaly is here specified to obtain an eastward propagating gravity wave. $\theta_{o}$ is chosen so that the initial density perturbation is zero. Unless stated otherwise the initial position of the perturbation is $x_{o}=10.000 \mathrm{~km}$ and its horizontal scale is $l_{p}=2000 \mathrm{~km}$. Its amplitude is $\delta h_{\max }=1 \mathrm{~m}$, small enough that the perturbation propagates as an eastward linear wave.

\subsection{Results for numerical experiments in adiabatic conditions}

Figure 3 shows results for the EP experiment. We see the propagation of the gravity wave signal ( $u, h, \theta$ fields) and the density perturbation created by the passage of the wave (here a pure advection of the density by the velocity perturbation) calculated by the numerical model. The numerical experiment (solving Eq. 3 and 4) is run for 150 days and on each plot we superimpose the signal structure every 10 days. The thick line on each plot is the analytical result (Eq. 8 and 11), giving the evolution of the maximum amplitude of the signal during the wave propagation. It is very close to the numerical results. Note that we have also plotted $-h$ instead of $h$ so as to represent the variations of the position of the thermocline, often represented by the $20{ }^{\circ} \mathrm{C}$ isotherm (and we thus define $\delta z 20=-h$ ). The wave is associated with a deepening of the thermocline, corresponding to a downwelling event.

The layer thickness, velocity and buoyancy propagate eastward with growing amplitude and the density perturbations monotonically increase eastward during the propagation of the wave, generating an EP type El Niño.

Figure 4 shows results for the $\mathrm{CP}$ experiment. Again the analytical results are close to the numerical ones, but in this case, the maximum density anomaly is reached in the middle of the basin (in the region of the back- 

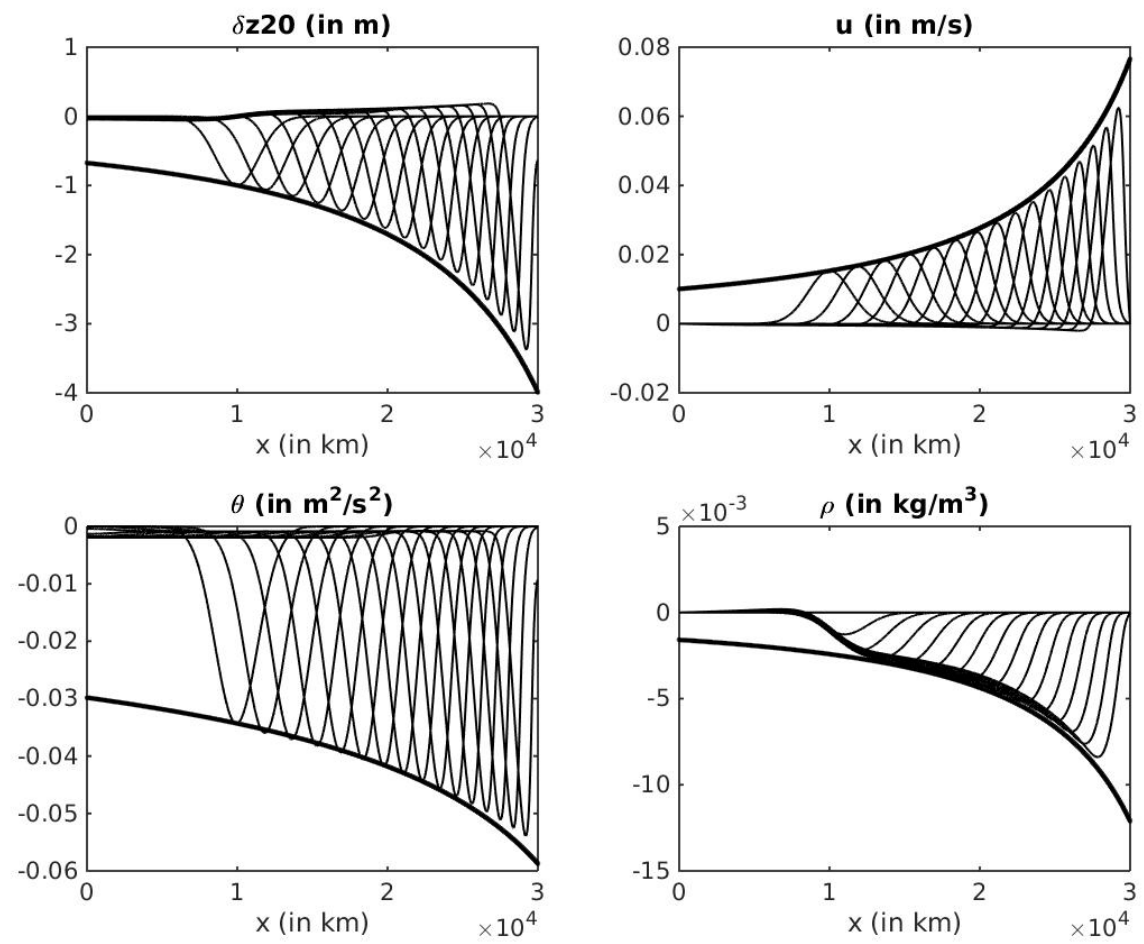

Figure 3: Evolution of the thermocline position $\delta z 20=-h$ representing the thermocline variations (top left), velocity anomaly (top right), $\theta$ (bottom left) and density anomaly (bottom right) for the EP experiment in adiabatic conditions for 150 days. In each panel, we superimpose the field output every 10 days. The solid thick curve indicates the analytical solution for the maximum perturbation created by the wave. The wave induces a downwelling and the creation of a negative density anomaly (or positive temperature anomaly). 
ground front), generating a CP type signal. Also note that the gravity wave signal ( $u, h, \theta$ fields) exhibit the same behaviour as before, with a monotonic increase of the wave amplitude at a rate that is almost twice the previous one.

\subsection{Physical analysis for adiabatic conditions}

To analyze the circumstances in which EP or CP types are reached, we discuss the analytical derivative of the density anomaly $\rho_{\max }^{\prime}$ (see Eq. 11):

$$
\rho_{\text {max }}^{\prime} \propto-\rho_{o}^{\prime \prime}+\frac{3}{4} \rho_{o}^{\prime}\left(\frac{\rho_{o}^{\prime}}{\rho_{o}}+\frac{H_{o}^{\prime}}{H_{o}}\right)
$$

For a CP type, the density anomaly reaches a maximum in the middle of the basin, so we must get $\rho_{\max }^{\prime}=0$ within the basin during the propagation.

For a configuration representative of the equatorial Pacific, the second term on the right hand side is always negative (the layer depth decreases $H_{o}^{\prime}<0$, the mixed layer density anomaly increases $\rho_{o}^{\prime}>0$ from West to East but $\left.\rho_{o}<0\right)$. Thus, if $\rho_{o}^{\prime \prime}$ is small, the perturbation of the density field monotonically increases eastward, yielding a structure for the density perturbation of the EP-type El Niño, as is the case in Fig. 3. To reach a CP type structure, $\rho_{o}^{\prime \prime}$ must be positive and sufficiently strong somewhere along the equator, a condition that requires a density front for the equilibrium state.

To conclude, for adiabatic evolution, the wave propagation is associated with a purely advective effect on the density field, and the physics is quite simple: the generation of EP or CP type only depends on the background stratification characteristics. The stronger the density gradient, the stronger the density anomaly created by a given velocity perturbation. Frontal regions are thus naturally subject to strong local anomaly generation and CP 

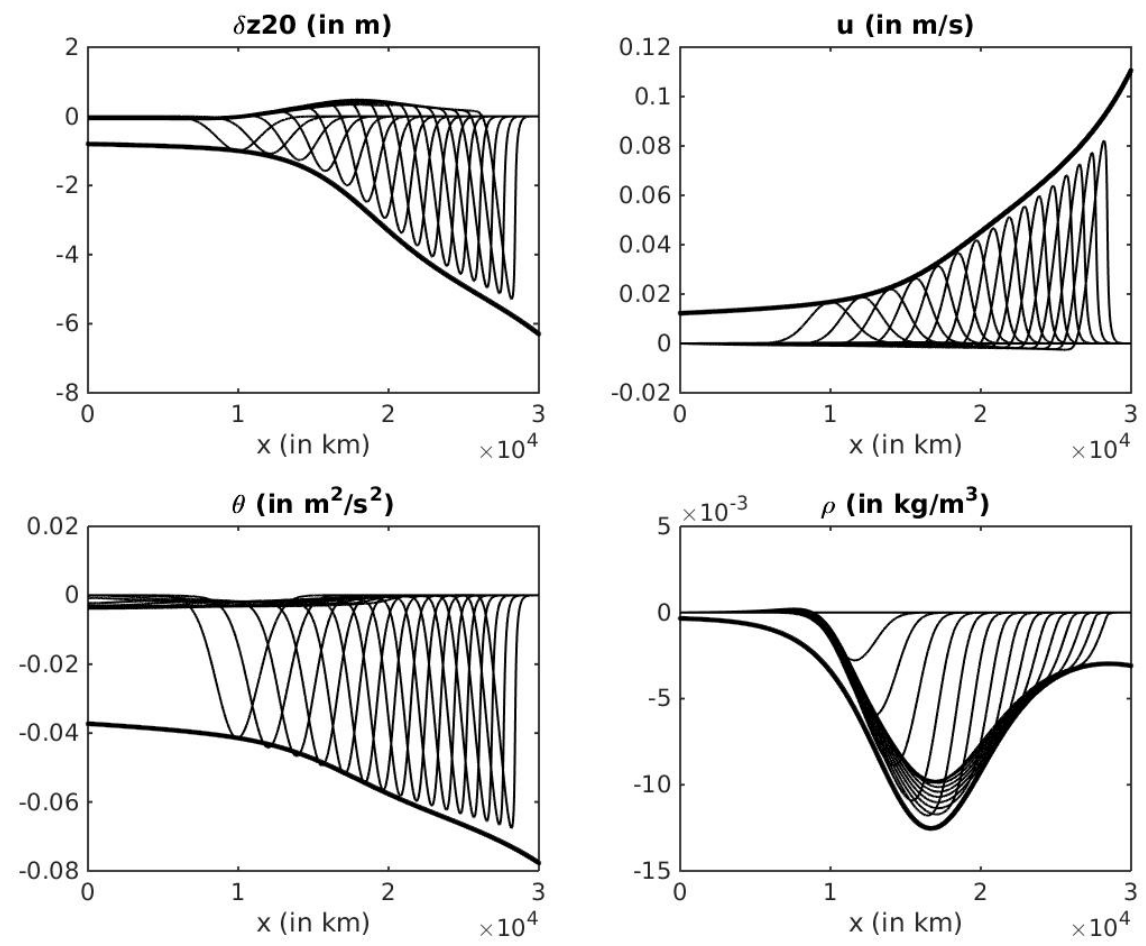

Figure 4: Same as Fig. 3 for the CP experiment. 
type structures. Note however that, away from the front, the perturbation amplitude continues to grow because of the decay of the background stratification. If this amplification process is strong enough, it is also possible to have a local maximum of the density anomaly (CP structure) followed by further amplification of the perturbation which reaches another local maximum near the coast.

\section{Results for linear waves in diabatic conditions}

\subsection{Parameterization of the forcing terms}

We now evaluate the influence of possible diabatic effects on the numerical and analytical results. For this, we repeat the EP and CP experiments presented in the previous section, but with diabatic forcing terms. If at equilibrium the latter are determined by the physical fields (see Eq. 4), in the general case they can be modified during the wave propagation. Two kinds of modifications can occur:

1. retroactive effects, due to ocean/atmosphere coupling and to the modification of the ocean forcings by the perturbations due to the gravity wave. For instance the modification of $H$ during the passage of the wave induces a modification of the wind stress forcing term;

2. external effects, associated with processes that have no link with the perturbations due to the gravity wave, and which can be considered as stochastic effects (e.g. stochastic wind bursts).

We only consider retroactive effects, which arise when forcing terms depend on the mixed layer characteristics. The variation of the forcing terms 
can then be written:

$$
\begin{aligned}
\delta F^{U} & =F\left(U_{o}+u, H_{o}+h, \Theta_{o}+\theta\right)-F_{o}\left(U_{o}, H_{o}, \Theta_{o}\right) \\
& \simeq \frac{\partial}{\partial U} F_{o} \cdot u+\frac{\partial}{\partial H} F_{o} \cdot h+\frac{\partial}{\partial \Theta} F_{o} \cdot \theta .
\end{aligned}
$$

$$
\begin{aligned}
\delta F^{U} & =-\alpha^{U} u, \\
\delta F^{H} & =-\alpha^{H} h, \\
\delta F^{\Theta} & =-\alpha^{\Theta} \theta .
\end{aligned}
$$

where the $\alpha$ values can thus be evaluated from existing parameterizations.

Note that more general parameterizations of the forcing terms including delocalized effects (e.g. a wind response located west of the density perturbations) are in principle possible but are beyond the scope of the present study.

\subsection{Analytical solutions for the wave signal in diabatic conditions}

The analytical solutions obtained under adiabatic conditions in section

3 can be extended to take into account Newtonian cooling diabatic terms

Eq. 17. We get (see Appendix B):

$$
\begin{aligned}
\theta & =\frac{C_{o}\left(x=x_{o}\right)^{1 / 2}}{C_{o}(x)^{1 / 2}} e^{-\left(\alpha^{\Theta}+\alpha^{U}\right) x / 2 C_{o}} \quad \theta_{o}(x / C(x)-t), \\
u & =\frac{\theta}{C_{o}(x)}=\frac{C_{o}\left(x=x_{o}\right)^{1 / 2}}{C_{o}(x)^{3 / 2}} e^{-\left(\alpha^{\Theta}+\alpha^{U}\right) x / 2 C_{o}} \theta_{o}(x / C(x)-t) \\
h & =\frac{\rho_{r e f} \theta}{g \rho_{o}(x)}=\frac{\rho_{r e f} C_{o}\left(x=x_{o}\right)^{1 / 2}}{g \rho_{o}(x) C_{o}(x)^{1 / 2}} e^{-\left(\alpha^{\Theta}+\alpha^{U}\right) x / 2 C_{o}} \theta_{o}(x / C(x)-t)(18)
\end{aligned}
$$


4.3. Analytical solutions for the density evolution in diabatic conditions

When taking into account diabatic terms, it is interesting to generalize the density evolution Eq. 6 and consider a damping term specifically depending on the surface density, leading to

$$
\partial_{t} \rho_{s}+U \partial_{x} \rho_{s}=\frac{\rho_{r e f}}{g H} F^{\Theta}-\frac{\rho_{s}}{H} F^{H}+F^{\rho}\left(\rho_{s}\right) .
$$

The additional term $F^{\rho}$ in the density equation plays a minor role and can be neglected for the physics associated with the propagating wave (Eq. 18 remain valid), but it is important for the density evolution itself. As already used above, we consider a small perturbation and linearize the function $F^{\rho}\left(\rho_{s}\right)$ to give

$$
\delta F^{\rho}=-\alpha^{\rho} \rho .
$$

Using Eq. 4, 17 and the existing relationship between $u, h, \theta$ for the wave signal (Eq. 18) yields the following linearized evolution equation for the surface density perturbation $\rho=\rho_{s}-\rho_{o}$ (see Appendix C):

$$
\partial_{t} \rho=-u \partial_{x} \rho_{o}+\frac{\rho_{r e f}\left(\alpha^{H}-\alpha^{\Theta}\right)}{g H_{o}} \theta-\alpha^{\rho} \rho .
$$

6 A new approximate solution is then derived (see Appendix C) for the maximum density perturbation:

$$
\rho_{\max } \propto\left[-\frac{\rho_{o}^{\prime}}{C_{o}^{3 / 2}}+\frac{\rho_{r e f}\left(\alpha_{o}^{H}-\alpha_{o}^{\Theta}\right)}{g H_{o} C_{o}^{1 / 2}}\right] e^{-\left(\alpha^{\Theta}+\alpha^{U}\right) x / 2 C(x)} e^{-\alpha^{\rho} l_{p} / C_{o}(x)} .
$$

This analytical solution looks quite complicated, but, as we will show, remains reasonably accurate. Notice that the $\alpha$ terms can either limit the generated density anomaly amplitude or increase it. In particular, the difference $\alpha^{H}-\alpha^{\Theta}$ determines the net effect $\left(\alpha^{H}-\alpha^{\Theta}<0\right.$ is associated with damping). 
Eq. 22 gives an analytical estimate of the maximum density perturbation generated by the passage of the gravity wave. At a given location, it is valid during the passage of the wave, but the $-\alpha^{\rho} \rho$ term continues to act after the passage of the wave: if $\alpha^{\rho}>0$ the density anomaly is then damped at a rate $e^{-\alpha^{\rho} t}$.

\subsection{Validation of the analytical solutions in diabatic conditions}

There are additional approximations in the derivation of the analytical solutions with diabatic terms (Eq. 18 and 22), but the solutions still remain quite accurate. We have indeed, tried many different combinations for $\alpha^{U}, \alpha^{H}, \alpha^{\Theta}, \alpha^{\rho}$ and have always found that the analytical predictions remained close to the numerical results (Eq. 3, 4, 17 and 20).

To illustrate this, we here resume the EP experiments (see Fig. 3), but with $\alpha^{U}=\alpha_{o}, \alpha^{H}=0, \alpha^{\Theta}=0$ and $\alpha^{\rho}=0$, where $\alpha_{o}=1 / 50$ days $^{-1}=$ $2.310^{-7} \mathrm{~s}^{-1}$ is a typical damping rate (as we will see below, this value corresponds to a threshold for the influence of diabatic effects). The numerical and analytical results are represented in Fig. 5. Note the analytical solution indeed gives good results. As can be expected, the velocity, buoyancy and layer depth anomalies are all damped in comparison with the adiabatic solution. The maximum density anomaly remains nearly constant during the eastward propagation. If $\alpha^{U}$ is further increased, the maximum density anomaly is reached close to the generation area and becomes of CP type (not shown).

Many other experiments have been performed and confirm the good agreement between the analytical and numerical model, even using complicated equilibrium states. To illustrate this, Fig. 6 shows the results for the $\mathrm{CP}$ configuration (see Fig. 4), but choosing $\alpha^{U}=\alpha^{\rho}=0, \alpha^{H}=0.3 \alpha_{o}$ 

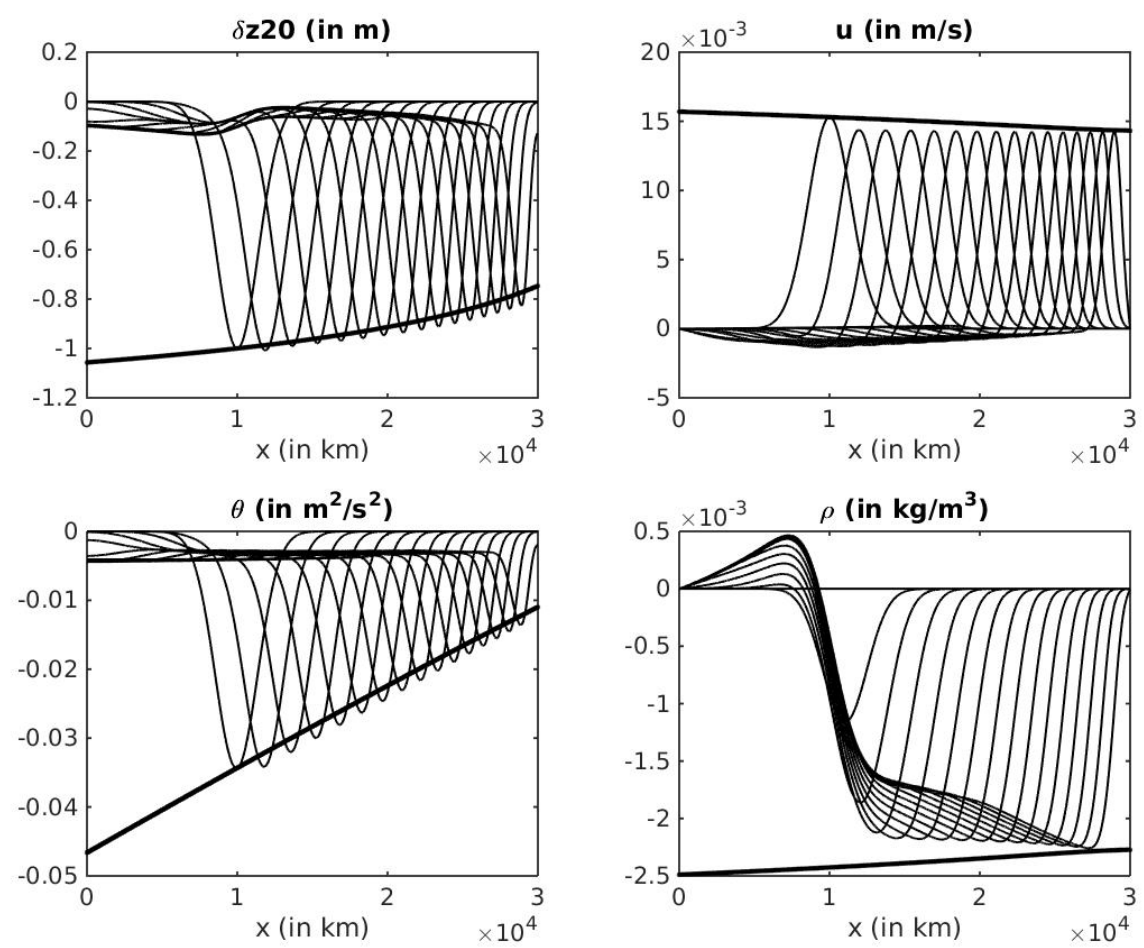

Figure 5: EP experiment with diabatic forcings $\left(\alpha^{U}=\alpha_{o}, \alpha^{H}=0, \alpha^{\Theta}=0, \alpha^{\rho}=0\right)$. The graph shows the same fields as Fig. 3. 
${ }_{419}$ and $\alpha^{\Theta}=-0.3 \alpha_{o}$. The choice for the parameter is arbitrary (in particular

442 (about $30 \%$ ) with other choices for the background characteristics $H_{o}$ and

$443 \rho_{o}$, the analytical model seems able to predict the density anomaly, even in 

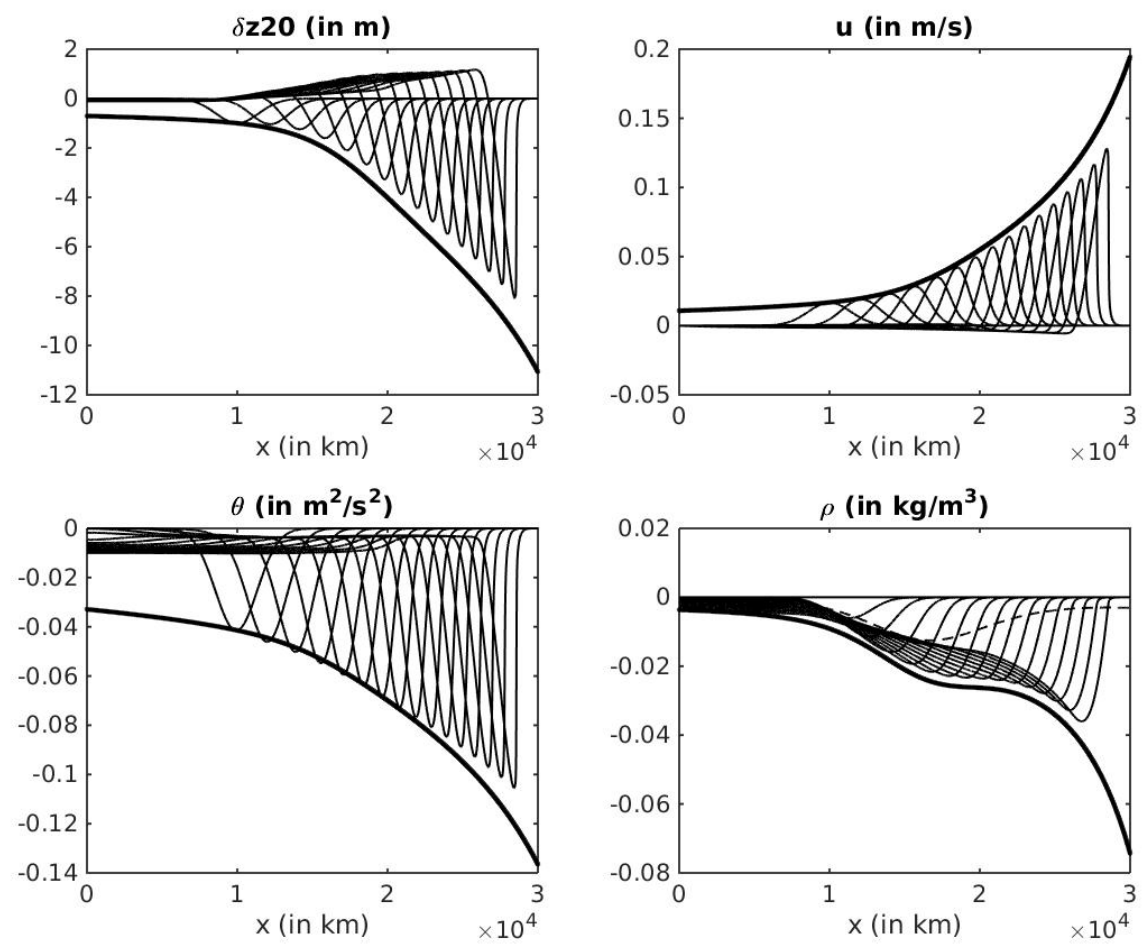

Figure 6: CP experiment with diabatic forcings $\left(\alpha^{U}=\alpha^{\rho}=0, \alpha^{H}=0.3 \alpha_{o}\right.$ and $\alpha^{\Theta}=$ $-0.3 \alpha_{o}$ ). The graph shows the same fields as Fig. 4. Note the similarity with Fig. 4 for the shape of the propagating anomalies, but the strong modification for the maximum density anomaly (the dashed line on the density plot represents the analytical solution valid without forcings). 
finite amplitude, non-linear regimes.

As far as the amplitude of the density perturbation is concerned, note that a density anomaly of $\delta \rho_{\max }=-1 \mathrm{~kg} / \mathrm{m}^{3}$ roughly corresponds to an anomaly of $4{ }^{\circ} \mathrm{C}$ and is thus taken as the order of magnitude representing strong impact. In the present case, the maximum anomaly reaches $\delta \rho_{\max }=$ $-0.25 \mathrm{~kg} / \mathrm{m}^{3}$ and remains modest. This is further accentuated by nonlinear effects as they generate damping of the wave in comparison with the linear theory. It thus appears that, to reach significant density anomalies, amplifying retroactive effects are necessary. Nevertheless, the conclusions on the localization of density anomalies from section 3 and 4 remain valid: in the present simplified configuration, nonlinearities do not modify the EP/CP patterns.

\subsection{Influence of diabatic retroactive effects}

We now estimate when retroactive effects, associated with the Newtonian cooling terms and the damping rates $\alpha$, have a significant effect on the structure (CP v.s. EP) and strength of the density anomaly generated by the wave. We thus use Eq. 22 to analyze the sensitivity of the density anomaly structure to the configuration parameters, in particular the damping rates. Fig. 9 shows the results for the EP configuration (see section 3.3 and Fig. 3 for the $\alpha=0$ adiabatic case) where we have set $\delta h_{\max }=20 \mathrm{~m}, \alpha^{\rho}=0$ and $\alpha^{H}=\alpha^{U}=\alpha^{\Theta}=\alpha$, where $\alpha \in\left[-2 \alpha_{o}, \quad+2 \alpha_{o}\right]\left(\alpha_{o}=1 / 50\right.$ days $\left.^{-1}=2.310^{-7} s^{-1}\right)$. For this choice, the structure of the density anomaly is of the EP type until retroactive processes are strongly damping. Indeed, when $\alpha>0.5 \alpha_{o}$ the rate of decrease is strong enough to counteract the amplifying effect of the modification of the stratification and the initial perturbation decreases. In this 

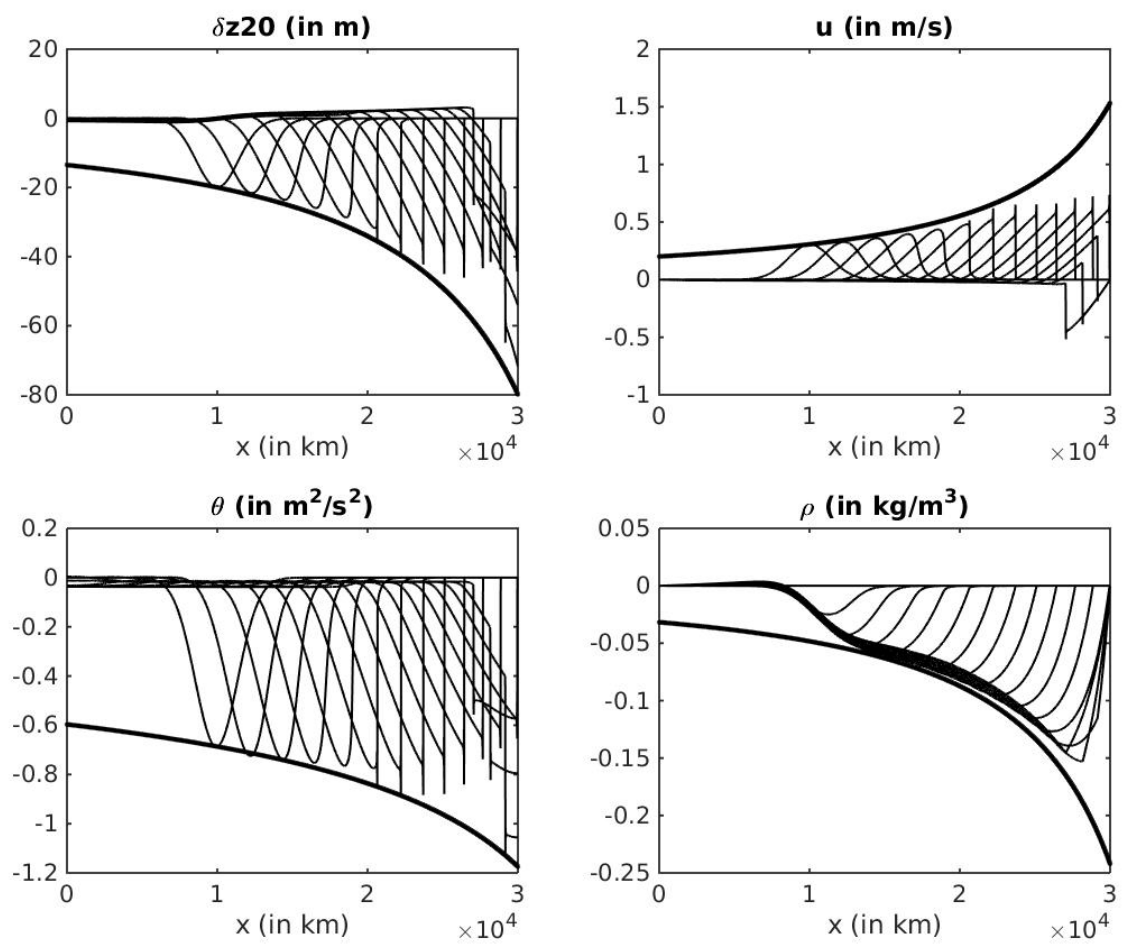

Figure 7: Same EP configuration as Fig. 3 except for $\delta h_{\max }=20 \mathrm{~m}$. Evolution of the layer depth anomaly (top left), velocity anomaly (top right), $\theta$ (bottom left) and density anomaly (bottom right) for 150 days with an output every 10 days. For each field, the solid thick curve indicates the analytical solution for the maximum perturbation created by the wave. 

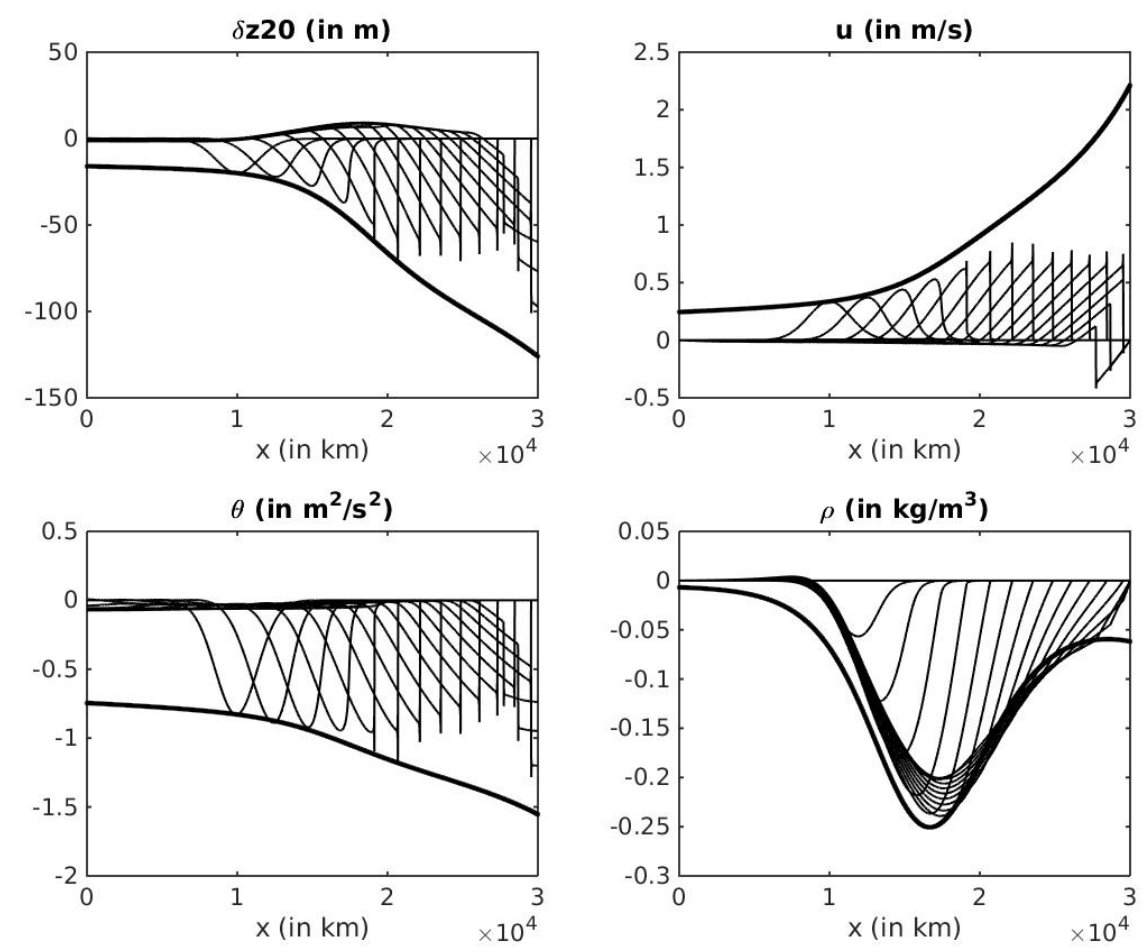

Figure 8: Same CP configuration as Fig. 4 except for $\delta h_{\max }=20 \mathrm{~m}$. Evolution of the layer depth anomaly (top left), velocity anomaly (top right), $\theta$ (bottom left) and density anomaly (bottom right) for 150 days with an output every 10 days. For each field, the solid thick curve indicates the analytical solution for the maximum perturbation created by the wave. 
case the structure becomes $\mathrm{CP}$, but note that the maximum perturbation values does not exceed $\delta \rho_{\max }=-0.05 \mathrm{~kg} / \mathrm{m}^{3}$ (for $\alpha \in\left[0.5 \alpha, 2 \alpha_{o}\right]$ ), which is weak, even for CP El Niño events. When the evolution corresponds to EP type $\left(\alpha<0.5 \alpha_{o}\right)$, the maximum values reached at the eastern coast remain modest $\left(\delta \rho_{\max }<-0.2 \mathrm{~kg} / \mathrm{m}^{3}\right.$ for $\alpha=0$ for instance) unless the retroactive processes are strongly amplifying: $\delta \rho_{\max }<-1 \mathrm{~kg} / \mathrm{m}^{3}$ for $\alpha=-0.5 \alpha_{o}$.

Fig. 10 shows the results for the $\mathrm{CP}$ configuration (see section 3.3 and Fig. 4 for the $\alpha=0$ adiabatic case), where we have again set $\delta h_{\max }=20 \mathrm{~m}$, $\alpha^{\rho}=0$ and $\alpha^{H}=\alpha^{U}=\alpha^{\Theta}=\alpha$, where $\alpha \in\left[-2 \alpha_{o},+2 \alpha_{o}\right]$. For damping situations $\left(\alpha \in\left[0,2 \alpha_{o}\right]\right)$, the structure remains of the CP type and the maximum density perturbation range is $\delta \rho_{\max } \in[-0.2,-0.1] \mathrm{kg} / \mathrm{m}^{3}$. Again, amplifying retroaction is necessary to reach strong enough density anomalies $\left(\delta \rho_{\max }<-0.4 \mathrm{~kg} / \mathrm{m}^{3}\right.$ when $\left.\alpha<-0.5 \alpha_{o}\right)$. When $\alpha<-0.6 \alpha_{o}$, the structure becomes EP, but for strongly negative $\alpha$, the amplitude of the density perturbation becomes unrealistically low : $\delta \rho_{\max }<-2 \mathrm{~kg} / \mathrm{m}^{3}$ for $\alpha<-\alpha_{o}$.

Changing the relationship between $\alpha^{\rho}, \alpha^{H}, \alpha^{U}$ and $\alpha^{\Theta}$ can modify the previous results qualitatively and quantitatively. We have tested several options and our results show that some general conclusion associated with the two previous configurations are robust:

- some amplifying retroaction ( $\alpha$ negative for some or all physical fields) is necessary to reach significant density (temperature) anomalies with a single wave (the case of multiple waves is addressed below);

- to reach a significant CP El Niño, the initial stratification plays a major role: the preexistence of a density front in the background stratification is necessary. 

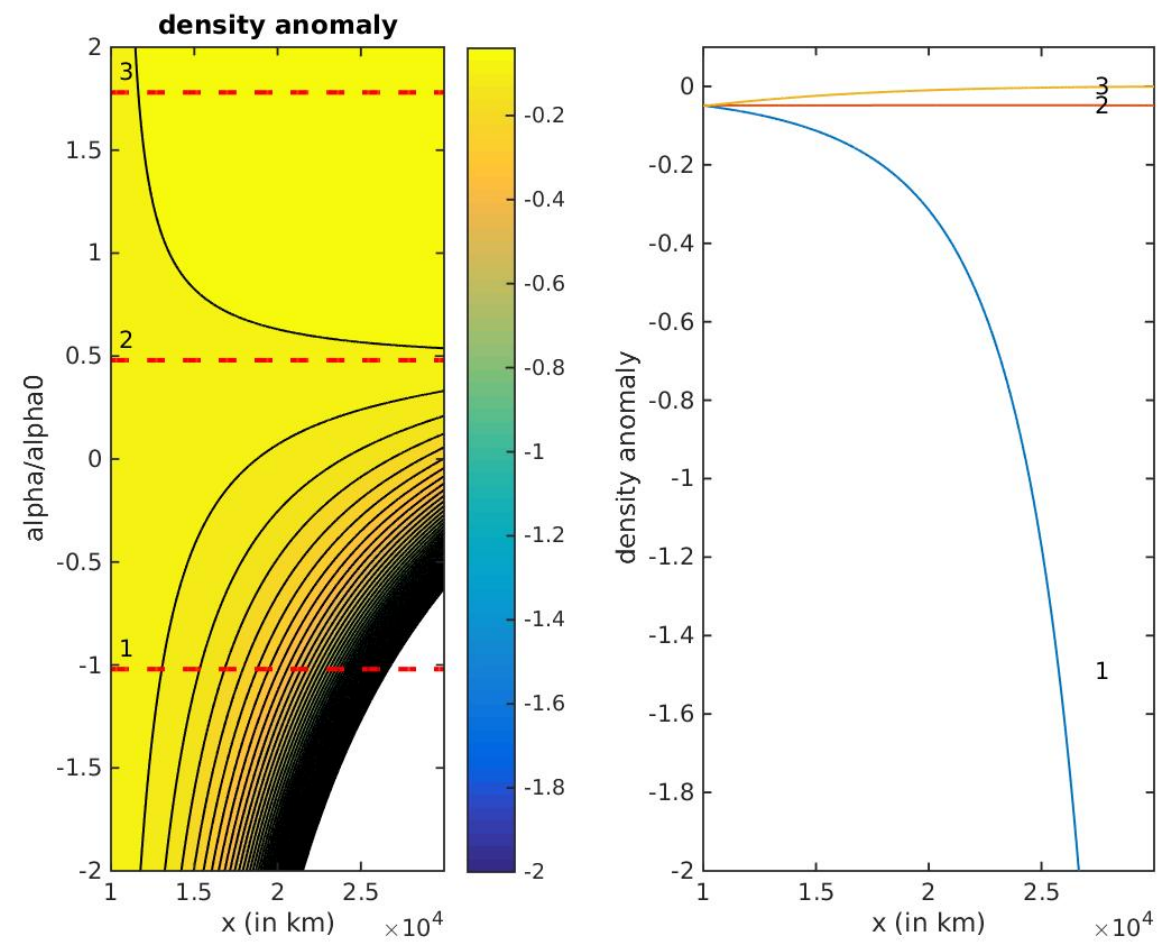

Figure 9: Left panel: density anomaly structure as a function of the zonal coordinate $x$ (in $\mathrm{km}$ ) and the damping rate $\alpha$ for the EP configuration (see section 3.3 and Fig. 3). Right panel: density anomaly structure as a function of the zonal coordinate $x$ (in $\mathrm{km}$ ) for selected damping coefficients (corresponding to the red dashed lines on the left panel). Note the transition from EP (1) to CP (3) and the intermediate structure with a flat density variation $(2)$. 
The evaluation of realistic ranges for the value of $\alpha$ can be determined from basic parameterizations of forcings and diabatic processes (see Barnier (1998) for a review). This is however beyond the scope of the present work and is left for a future study.

\subsection{Effect of the perturbation scale}

We here vary the initial perturbation scale $l_{p}$ from $1000 \mathrm{~km}$ to $6000 \mathrm{~km}$ (cf Eq. 14, $l_{p}=2000 \mathrm{~km}$ in all previous experiment). All damping rates are set to zero and we calculate the density anomaly for both the EP (see Fig. 3 ) and CP (see Fig. 4) configurations but with $\delta h_{\max }=20 \mathrm{~m}$. As can be expected, the larger the perturbation scale, the stronger the maximum density anomaly and of course the wider the area where strong density anomalies are created. The structure (EP or CP) of the perturbation is not modified and we notice that the maximum density anomalies remain modest unless $l_{p}$ becomes very large: $\delta \rho_{\max }<-0.7 \mathrm{~kg} / \mathrm{m}^{3}$ for $l_{p}>6000 \mathrm{~km}$. We thus believe that varying the initial perturbation scale is not enough to reach the 1 values associated with the strongest El Niño events and that, as already un2 derlined above, multiple waves or significant amplifying retroaction effects are necessary.

\subsection{Effect of the background circulation}

The effect of the background circulation $U_{o}$ was set to zero in all previous analytical and numerical results. We were not able to find a solution when $U_{o} \neq 0$ with our analytical framework, but we have evaluated the effect of 8 a background shear using the numerical simulations. We have chosen :

$$
U_{o}(x)=U_{\max } 0.5\left(1-\cos \left(\frac{2 \pi x}{L}\right)\right)
$$



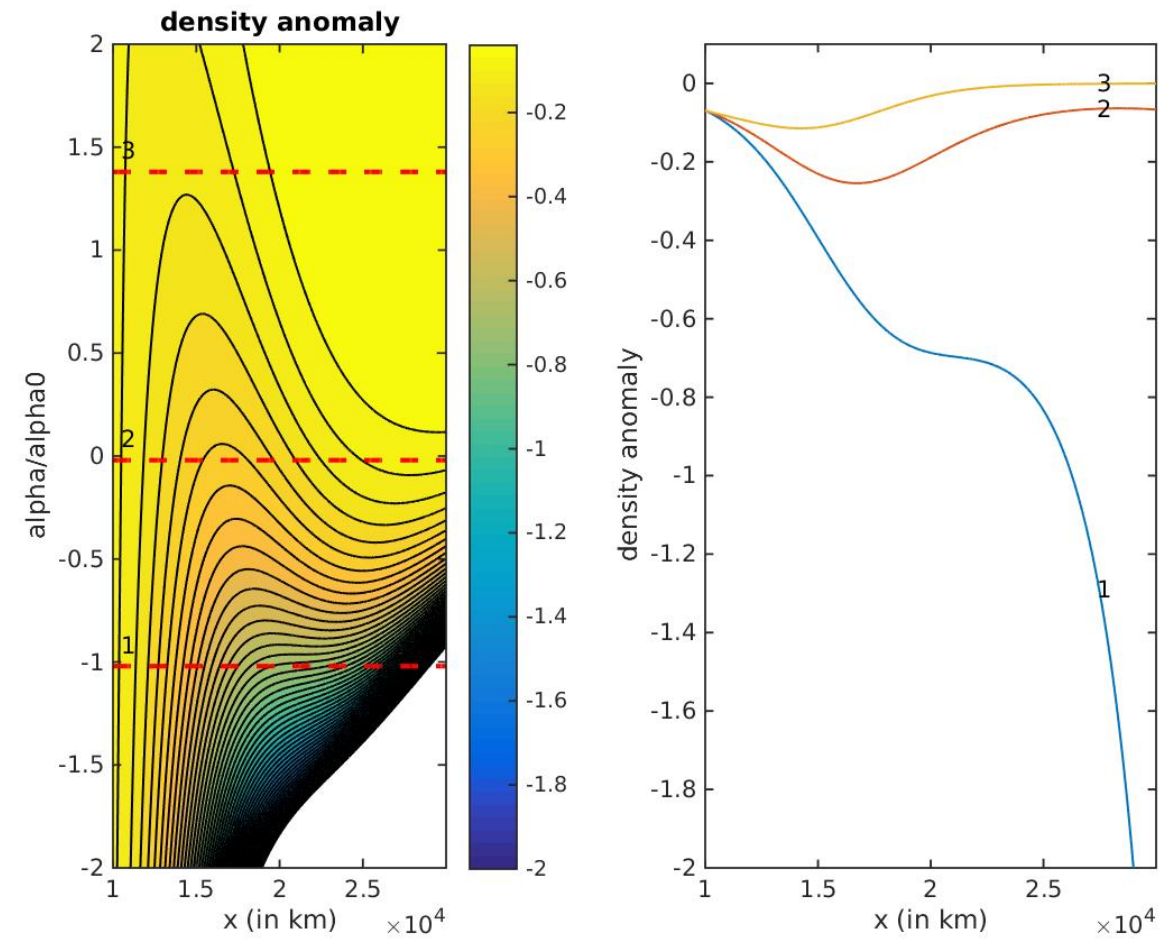

Figure 10: Left panel: density anomaly structure as a function of the zonal coordinate $x$ (in $\mathrm{km}$ ) and the damping rate $\alpha$ for the CP configuration (see section 3.3 and Fig. 4). Right panel: density anomaly structure as a function of the zonal coordinate $x$ (in $\mathrm{km}$ ) for selected damping coefficients (corresponding to the red dashed lines on the left panel). Note the transition from CP (2 and 3$)$ to EP (1). 

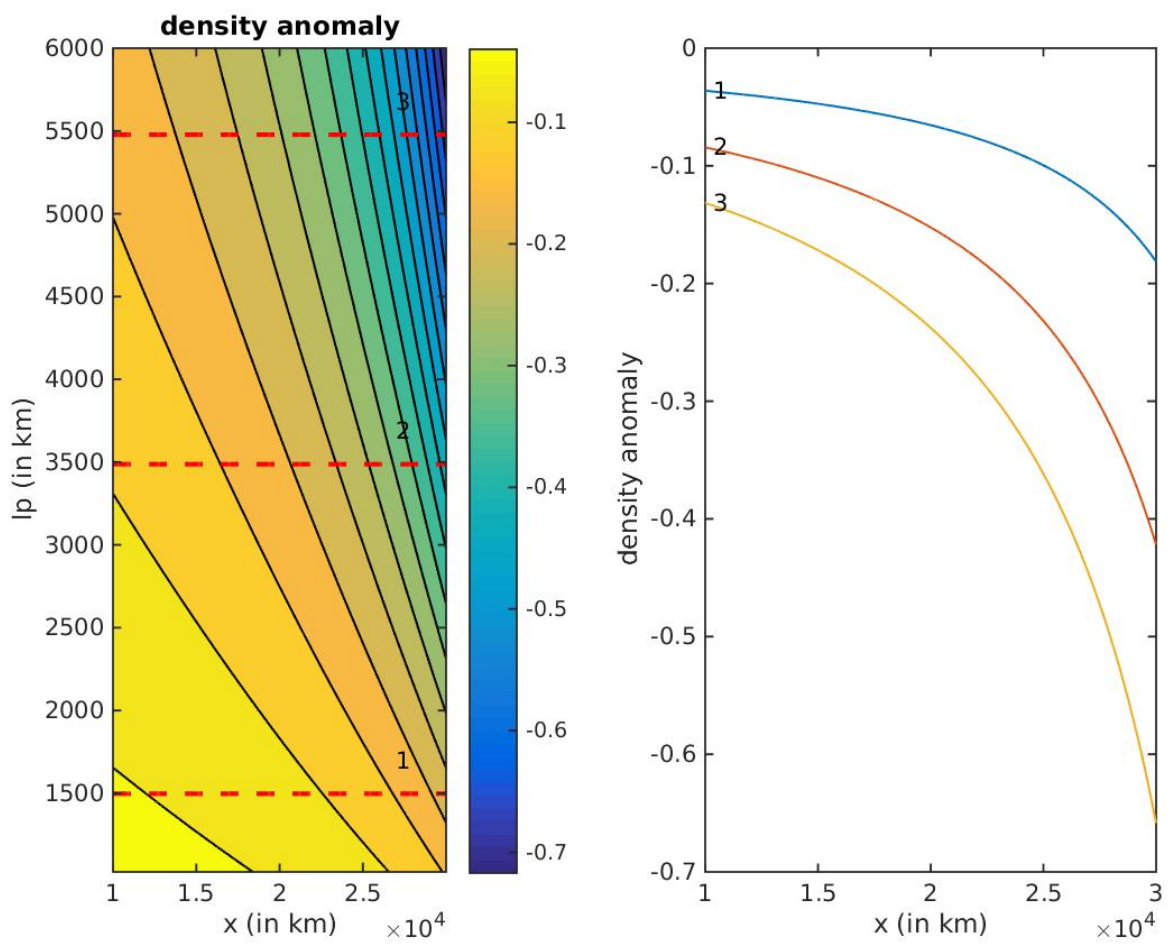

Figure 11: Left panel: density anomaly structure as a function of the zonal coordinate $x$ (in $\mathrm{km}$ ) and the initial -Gaussian- perturbation scale $l_{p}$ for the EP configuration (see section 3.3 and Fig. 3). Right panel: density anomaly structure as a function of the zonal coordinate $x$ (in $\mathrm{km}$ ) for selected lengthscales (corresponding to the red dashed lines on the left panel). Note the structure remains EP but the density anomaly increases with the perturbation lengthscale. 

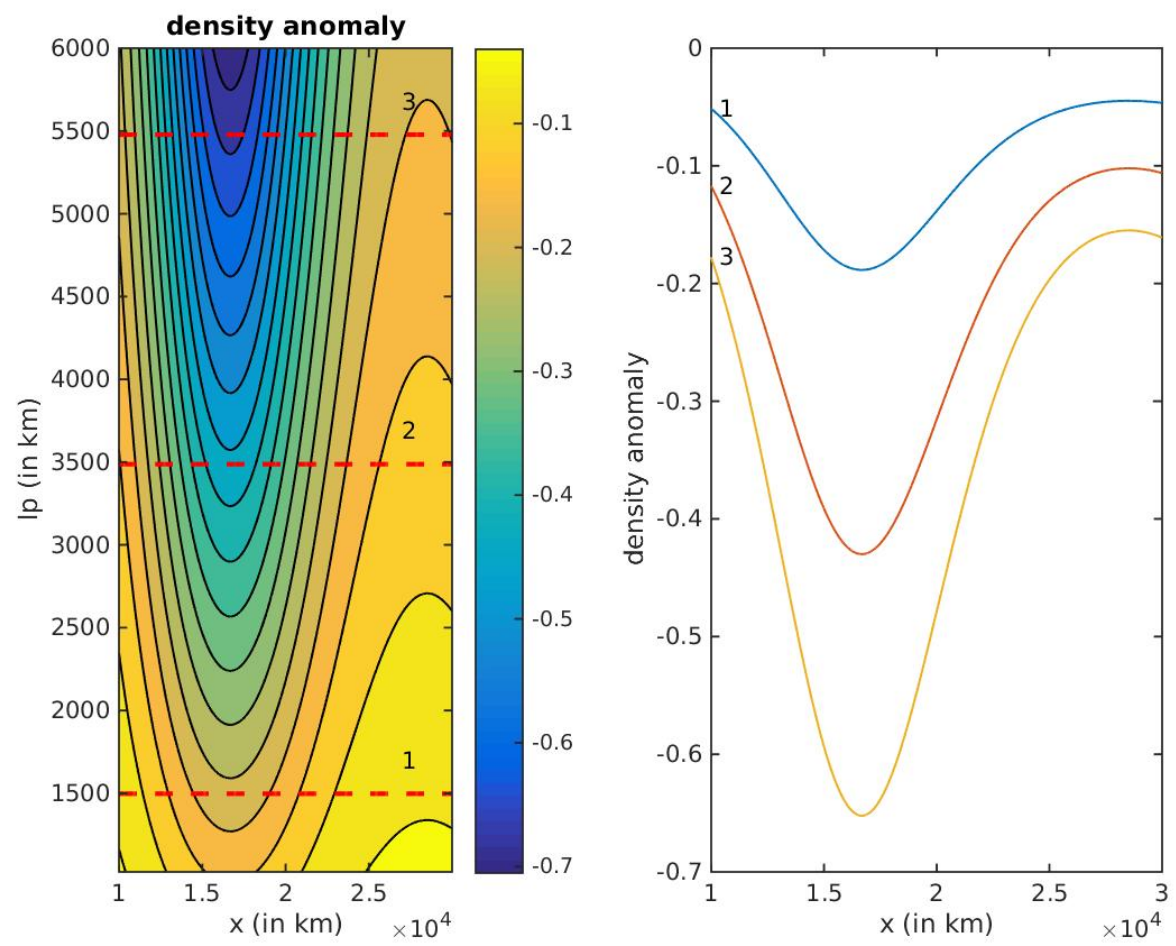

Figure 12: Left panel: density anomaly structure as a function of the zonal coordinate $x$ (in $\mathrm{km}$ ) and the initial -Gaussian- perturbation scale $l_{p}$ for the CP configuration (see section 3.3 and Fig. 4). Right panel: density anomaly structure as a function of the zonal coordinate $x$ (in $\mathrm{km}$ ) for selected lengthscales (corresponding to the red dashed lines on the left panel). Note the structure remains CP but the density anomaly increases with the perturbation lengthscale. 
so that $U_{o}$ satisfies the closed boundary conditions at $x=0$ and $x=L$ (extension of the domain). $U_{\max }$ is the maximum background velocity, reached at the center of the domain.

We have repeated the reference EP and $\mathrm{CP}$ experiments, but with a background velocity. The density evolution pattern is modified by the additional advection, but we have found that our previous results remain valid as long as the maximum background velocity $U_{\max } \in[-0.5 \mathrm{~m} / \mathrm{s}, 0.5 \mathrm{~m} / \mathrm{s}]$. Observations indicate that the mean velocities along the equator are in this range (see Picaut et al., 1997). In fact, the WKB approximation (see Gill (1982), chapter 8)) shows that, if the background velocity gradient is small, the evolution of a perturbation (in particular its amplitude) is not modified by the background current. There is just a shift in the wave group velocity: $C_{g}^{U}=C_{g}^{U=0}+U$. However, special attention must be paid to the density anomaly since it is not a wave propagation signal. Also, note that since $F^{H} \neq 0$ (see Eq. 4 ), there exists a diabatic term in the density anomaly evolution equation (see Eq. 6), and for a westward background flow $\left(U_{\max }<0\right)$ the density anomaly is now damped on the eastern side and amplified on the western side. Adding damping effects probably also complicates the physics. Further studies are obviously needed to evaluate the limits and compare with realistic fields.

\section{Effect of multiple waves: from CP to EP El Niño}

It is interesting to note that in the $\mathrm{CP}$ configuration, in the presence of a background density front, the effect of the wave is to smooth the front. Figure 13a shows the evolution of the total upper layer density $\rho_{s}$ for the CP case and $\delta h_{\max }=20 \mathrm{~m}$ (same experiment as in Fig. 8) and clearly shows 
the smoothing effect. We may thus wonder if multiple waves on an initially CP configuration could eventually yield a structure that would favor an EP configuration.

To test this idea, we have used the numerical model with a specific background condition. The basin scale has been reduced to $L=17.000 \mathrm{~km}$ (representative of the equatorial Pacific zonal length) and the background density structure is given by Eq. 13 with $\Delta \rho_{\max }^{\text {lin }}=2.5 \mathrm{~kg} / \mathrm{m}^{3}, \Delta \rho_{\max }^{\text {th }}=$ $1 \mathrm{~kg} / \mathrm{m}^{3}$ and $L_{t h}=1.000 \mathrm{~km}$, and the front position is located at $x_{t h}=$ $3.000 \mathrm{~km}$. The background mixed layer depth structure has also been modified, and we have chosen:

$$
H_{o}=H_{\text {mean }}-\Delta H_{\max } \frac{x-L / 2}{L}-\Delta H_{\max }^{t h} t h\left(\left(x-x_{t h}\right) / L_{t h}\right) .
$$

with $H_{\text {mean }}=115 \mathrm{~m}, \Delta H_{\max }=70 \mathrm{~m}$ and $\Delta H_{\max }^{\text {th }}=30$. The initial perturbation located at the western coast $\left(x_{o}=0 \mathrm{~km}\right)$, its extension is $l_{p}=3000 \mathrm{~km}$ and $h_{\max }=20 \mathrm{~m}$. Six waves of this type are generated (one per month for 6 months). The boundary conditions have been arranged so that the wave is radiated (no amplification by bouncing at the eastern boundary). Finally, the damping rates have been set to zero, except for $\alpha^{\rho}=0.15 \alpha_{o} \simeq 3.510^{-8} s^{-1}$ (corresponding to a damping time scale of about one year). Figure 13b-c shows the evolution of the mixed layer density and height anomalies for 300 days. Note the displacement of the maximum density anomaly, from the center of the basin for the first waves to the eastern coast for the last wave. The strength of the density anomaly also reaches $\delta \rho_{\max } \simeq-1 \mathrm{~kg} / \mathrm{m}^{3}$, which is the typical value of a strong El Niño 6 event. This result illustrates the possibility of progression from CP to EP El Niño. 

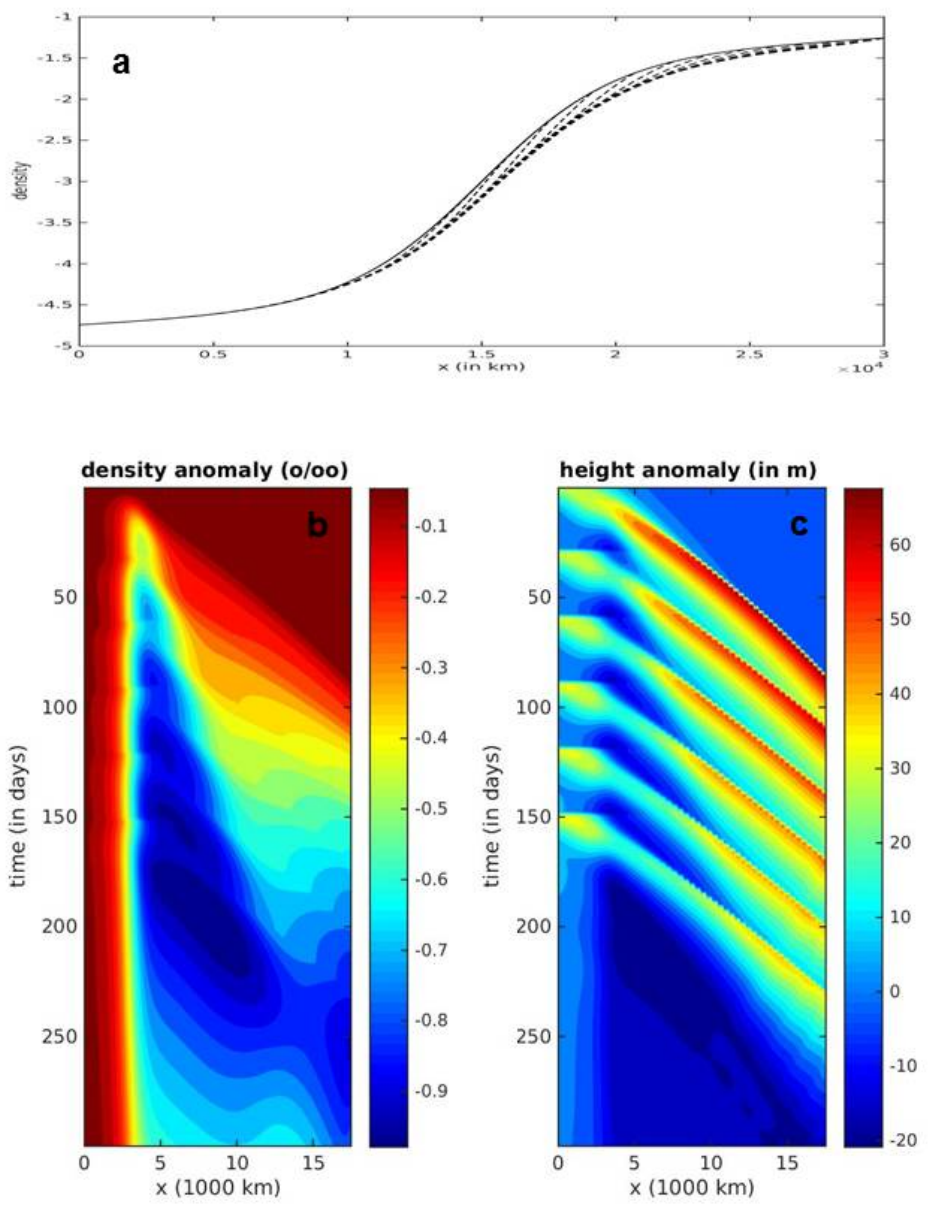

38

Figure 13: (a) Evolution of the total upper layer density structure for a 150 days with an output every 10 days (initial state in plain, evolution in dashed lines). Evolution of the density structure (b) and mixed layer depth (c) for 300 days for a simulation with multiple equatorial waves (6 waves launched every month for 6 months). 


\section{Discussion}

In the present work, our goal was to understand the mechanisms leading to the localization of El Niño events either in the Central (CP) or Eastern Pacific (EP) and their continuity. We have thus presented a simplified model to study the effect of equatorial Kelvin waves on the generation of density (temperature) anomalies. We considered the evolution of a reduced gravity, mixed layer along the equator and the equations are thus 1D. Approximate analytical solutions have been found for linear waves propagating in a spatially variable background stratification, maintained by diabatic forcings. Damping/amplifying effects, associated with retroactive processes, have been taken into account in the form of Newtonian cooling terms. The non-linear equations have been solved numerically to validate the theoretical results.

We have first studied adiabatic cases for which there is no feedback of the perturbation of the wave on the forcings (section 3 ). In this case the density (or temperature) field is purely advected by velocity perturbation associated with the wave during its passage. The final density anomaly depends on the amplitude of the displacement and on the local density gradient. Its shape is given by Eq. 11.

Figure 14 schematizes the evolution of the wave and temperature field in this adiabatic case (here we have used temperature instead of density for easier connection with the usual ENSO description). The propagation speed of the Kelvin wave diminishes eastward (blue arrows) so the perturbation height (dashed blue lines) and velocity (red arrows) increase eastward leading to greater displacement of isotherms (vertical red lines) and stronger anomaly at the Eastern boundary. This process favors EP events (Fig. 14, 
left column). Strong local density gradients, associated with fronts, generate strong local anomalies. Fronts are also associated with local deceleration of the wave propagation, leading to stronger local amplification of the wave perturbation (height and velocity). Both processes favor the generation of stronger density anomalies in the vicinity of the initial front, corresponding to a $\mathrm{CP}$ event (Fig. 14, right column).

In this adiabatic case, the existence of a front is thus a prerequisite to getting a CP structure. However, as shown by Eq. 11 and 15 and schematized in Fig. 14, the type of the final structure depends on details of the initial stratification. There thus exists a continuity between $\mathrm{CP}$ and EP events whose occurrence depends on the shape of the background surface density or layer depth. Table 1 indicates how several parameters influence the structure type if all other parameters are kept constant. For instance, according to our results, a shallower mean upper layer depth (diminishing $\overline{H_{o}}$ ) or a stronger difference between western and eastern upper layer depths $\left(\partial_{x} H_{o}\right.$ becomes "more negative") favors EP structures. Conversely, increasing the mean upper layer temperature (so that the mean density $\overline{\rho_{o}}$ becomes "more negative") or a weaker difference between western and eastern upper layer temperature (so that $\partial_{x} \rho_{o}$ becomes closer to 0 ) favors CP structures. These rules have to be used cautiously as in practice the parameters discussed in table 1 do not evolve independently.

It has also been shown that retroactive effects, associated with damping or amplification of the wave perturbations, or a succession of multiple waves are also important factors influencing the fate of an ENSO. Again table 1 summarizes the influence of these parameters. Amplifying feedbacks favor $\mathrm{EP}$, and it is possible to change a $\mathrm{CP}$ event into a EP event, provided am- 
EP

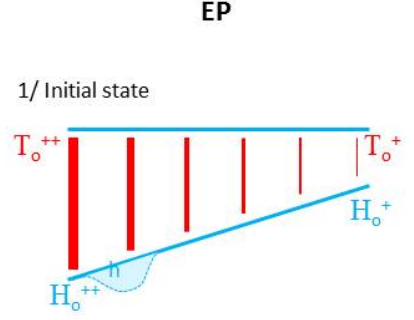

2/ Propagation phase

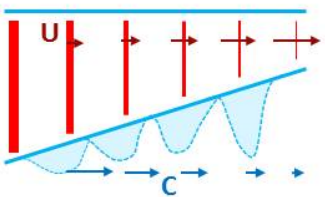

3/ Final state

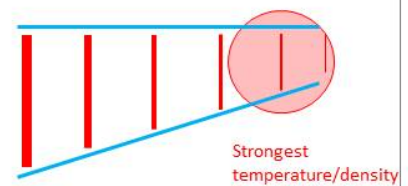

anomaly at east
CP
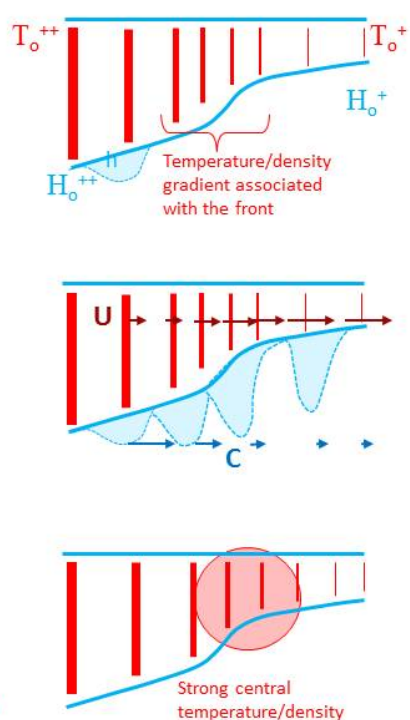

anomaly created by

displacement of the front

Figure 14: Schematic vertical sections illustrating the impact of a Kelvin wave on the upper-layer temperature field along the equator, in adiabatic conditions. Thick blue lines denote the surface and base of the layer, thin dashed lines denote the layer thickness perturbation associated with the Kelvin wave, whose propagation speed diminishes eastward (blue arrows). The dark red arrows show the Kelvin wave current perturbation and increase eastward. The red vertical lines correspond to isotherms, the thicker the line, the higher the temperature. The temperature field is purely advected by the velocity perturbation due to the Kelvin wave. The final anomaly depends on the displacement amplitude and on the initial local gradient of temperature. The strongest anomaly can be reached at the center (corresponding to a $\mathrm{CP}$ event) if there is a temperature front (right column). 


\begin{tabular}{|c|c|c|c|c|c|c|}
\hline \multirow{2}{*}{\multicolumn{2}{|c|}{\begin{tabular}{|l|} 
Parameters/values \\
$-\partial_{x 2} \rho_{\circ}$ (front)
\end{tabular}}} & \multicolumn{5}{|l|}{ small } \\
\hline & & (0) & EP & & & CP $\quad(+\infty)$ \\
\hline \multirow{2}{*}{$\mathrm{H}_{\mathrm{o}}$} & $\begin{array}{l}\mathrm{H}_{0}(\text { mean } \\
\text { value })\end{array}$ & (0) & EP & & & CP $\quad(+\infty)$ \\
\hline & $\partial_{x} H_{0}$ & $(-\infty)$ & EP & & & (0) \\
\hline \multirow{2}{*}{$\rho_{\circ}$} & $\begin{array}{l}\bar{p}_{\mathrm{o}} \text { (mean } \\
\text { value) }\end{array}$ & $(-\infty)$ & CP & & & (0) \\
\hline & $\partial_{x} \rho_{0}$ & (0) & $\mathrm{CP}$ & & & $(+\infty)$ \\
\hline \multirow{2}{*}{\multicolumn{2}{|c|}{ Number of waves }} & (1) & EP & & & $(+\infty)$ \\
\hline & & (1) & CP & & & EP $\quad(+\infty)$ \\
\hline \multirow{2}{*}{\multicolumn{2}{|c|}{$\alpha$}} & $(-\infty)$ EP & $\operatorname{EP}(<0)$ & \multirow{2}{*}{$\begin{array}{l}\text { Weak } \\
\text { influence }\end{array}$} & (>0) EP & $\mathrm{CP}(+\infty)$ \\
\hline & & $(-\infty)$ EP & $C P(<0)$ & & $(>0) C P$ & $\mathrm{CP}(+\infty)$ \\
\hline
\end{tabular}

Table 1: Diagram indicating how parameters influence the structure type (CP or EP). The important parameters are $\partial_{x^{2}} \rho_{o}$ (indicating the strength of the density front), the mean values and gradients of the upper layer depth and density $\left(\overline{H_{o}}, \partial_{x} H_{o}, \overline{\rho_{o}}, \partial_{x} \rho_{o}\right)$, the number of successive Kelvin waves and the effect of diabatic feedbacks, parameterized as Newtonian cooling terms $\alpha(\alpha<0$ cort2sponds to amplifying feedbacks, $\alpha>0$ to damping). The specific range of evolution (small values to high values) for each parameter is given in parenthesis. 
plification is strong enough. Damping feedbacks favor CP structures, and it is possible to transform an EP into a $\mathrm{CP}$ structure with strong enough damping. The thresholds beyond which the density structure is strongly modified, as a function of all parameters can be evaluated using our analytical results. The number of waves does not modify the fate of an EP event but can transform an initially $\mathrm{CP}$ into an EP event. This result is consistent with observations that have shown that multiple westerly wind bursts are necessary to get an EP El Niño (see Menkes et al., 2014). The number of equatorial waves necessary to switch from CP to EP depends on the details of the initial background stratification and wave characteristics, so more studies are needed to fully understand the switching mechanism. This is left for future work and here we merely illustrated this possibility.

The effects of non-linearities or of a background zonal current have been studied numerically and we found they have little impact on our results. However, in nature, non-linear evolution can also involve energy transfer to higher vertical modes (Cravatte et al., 2003), a process that was not evaluated in the present framework with one-layer model. Finally, we have also shown that the anomalies that are created generally remain modest so to obtain strong enough anomalies, there must either be strong retroactive amplification or a succession of waves whose effect accumulates.

Our results describe several aspects of the evolution of an equatorial Kelvin wave and of its impact on the upper layer density field. It provides an explanation of the continuity between CP and EP El Niño events. However, this is obviously subject to some caution, as the process oriented configuration is very simple and the model relies on many assumptions: vertically homogeneous upper (mixed) layer; reduced gravity; validity of the 1D configuration; constant lower layer density reference; no meridional velocity 
and no background zonal velocity (for the analytical solutions); existence of a mean background state (stratification), maintained by mean forcings. We have also linearized the dynamics for the analytical calculations, and for the retroactive effect of the wave perturbation which has been parameterized in the form of Newtonian cooling. The ability to switch from CP to EP types with multiple waves relies on the choice of the initial perturbation and background structure, so that we cannot claim this result is general. Finally, let us again mention that this study focuses on the effect of equatorial Kelvin waves, once they have been triggered by some unbalancing of the mean state, for instance by anomalous westerly wind bursts in the western part of the basin. We have not studied the mechanisms responsible for this triggering, which is also major ingredient of the ENSO process, as it determines the shape and strength of the initial Kelvin wave.

A number of complementary studies can follow from the present results. First it should be possible to take into account two tracer equations, one for salt and one for temperature, and evaluate the effect of salinity on the previous results. It may also be possible to extend the analytical model to take into account a mean background zonal velocity. At least, as mentioned above, a thorough numerical study could be performed to evaluate more precisely the effect of the background advection. The influence of multiple waves is also a subject of great interest. Previous studies have shown that the number of Kelvin waves generated by westerly wind bursts is a key factor in determining the strength and final structure of the temperature anomaly (see (Menkes et al., 2014)). Admitting that the initial structure generated by a primary Kelvin wave is of the CP type, the present model can help evaluate the number, frequency and strength of individual waves to reach an EP El Niño, including the combination of upwelling and downwelling 
Kelvin waves (Su et al., 2018).

Another possibility is to test the effect of meridional variations, which could play a significant role in the generation of strong EP events (Chen et al., 2016). It may be difficult to generalize the analytical model to a full $2 \mathrm{D}$ configuration, but simplified solutions (truncated to the first parabolic cylinder functions, see Thual et al., 2016) may be derived to account for 2D effects. Alternatively, it is possible to develop a $2 \mathrm{D}$ version of the reduced gravity numerical model (see Appendix A).

A further promising and important study is to compare the present formalism to realistic fields. This can be easily done for the adiabatic version of the model, but if, as expected, diabatic effects are important, one has to estimate the Newtonian cooling parameters $\alpha$ used in the present theory. Diabatic retroactive effects are associated with ocean/atmosphere coupling or mixing processes which depend on the stratification and velocity of the upper layer of the ocean. The latter are indeed modified during the propagation of the equatorial Kelvin wave, which modifies the diabatic fluxes. Simple parameterizations exists (see (Barnier, 1998)) that can be used to evaluate ranges for the variations of the $\alpha$ parameters. Preliminary considerations (not shown here) reveal that the value chosen for $\alpha_{o}$ in this article is of the right order of magnitude. Other processes, not represented in the present simplified model and not associated with ocean/atmosphere coupling or mixing processes, have been shown to play a role in the development of El Niño events. These include for example the transfer of energy to higher vertical modes or vertical advection of temperature/density anomalies into the upper mixed layer (Cravatte et al., 2003; Dewitte et al., 2012, 2013), or other 3D effects which are not properly represented in the present simplified configuration. We think most of the latter processes, can be crudely ap- 
proximated by Newtonian cooling terms in our model, but their associated $\alpha$ coefficients may be more difficult to evaluate.

As well as being involved in feedbacks, diabatic processes can also be important independently of the equatorial Kelvin wave perturbations. Stochastics events act as additional forcing terms which do not depend on the perturbation characteristics and have been sometimes identified as determinant for the development of strong EP El Niños (Fedorov et al., 2015). Even if the chosen form in Eq. 17 (a Newtonian damping/amplification) is very specific, in practice any effect having some influence on the physical characteristics of the perturbation can be represented by an appropriate choice of $\alpha$. For example, a negative $\alpha^{\Theta}$ can account for an additional heating event associated with an external process. Thus, even stochastic events could be fitted to the present framework and it would be possible to evaluate whether they can modify the fate (CP or EP and amplitude) of an El Niño event.

Evaluating the effect of climate change on the ratio between $\mathrm{CP}$ and EP types is obviously of great interest. Indeed, several oceanic parameters have been modified in the equatorial Pacific. The mean zonal gradient of SST has increased in the recent decades as a result of the strengthening of the Walker circulation (Sohn et al., 2013; England et al., 2014; Karnauskas et al., 2009). Moreover, while an increased ocean stratification and a flatter thermocline are predicted in GCM global warming scenarios (Timmerman et al., 1999; Yeh et al., 2009), the decades after the 1990s have been associated with a sharper thermocline and an increased La Niña-like background pattern (Xiang et al., 2013). The effect of climate change on El Niño events is still debated (see for instance Giese and Sulagna, 2011), but some observations show that more CP events occurred in recent decades (Yeh et al., 2009; Cai et al., 2014). Several studies have related such an increase of 
$\mathrm{CP}$ events to a change in the mean oceanic structure in the equatorial $\mathrm{Pa}$ cific. Using GCM experiments, Choi et al. (2011) link the increased CP occurence to a stronger zonal gradient of mean surface temperature, while Dewitte et al. (2012) stress changes in the central Pacific vertical stratification. The present study shows that if a stronger mid basin density front (parameter $\partial_{x}^{2} \rho_{o}$ in Table 1) favors CP events, other parameters have a nontrivial influence on the El Niño flavor. For example, an increased global zonal gradient of density/SST in the equatorial Pacific (parameter $\partial_{x} \rho_{o}$ in Table 1) or a shallower thermocline (parameter $\bar{H}_{o}$ ) favors EP events. Our numerical results (see section 6) underline that feedback effects or the number, strength or frequency of Kelvin waves, associated with the westerly wind bursts are also important (see Table 1). The latter effects are associated with ocean/atmosphere interactions, not directly represented in the analytical model, which can also evolve in a changing climate. To our knowledge, previous studies analyzing the effect of climate change on the observed evolution of El Niño flavor over recent decades have focused on only a few parameters. Our results advocate for a study involving the combined effects of all parameters identified in Table 1.

Finally, the present results show that knowledge of the details of the background stratification and of ocean/atmosphere fluxes are both necessary to be able to understand and predict the fate of an ENSO event. This advocates for the maintenance of a dedicated in situ observation system for oceanic and atmospheric measurements, which is currently being discussed in the framework of the TPOS2020 project (Tropical Pacific Observing System, http://tpos2020.org/). 
Acknowledgments. This study has been funded by CNES (french space agency; project TOSCA/OSTST "Alti-ETAO") and the program "IDEX attractivity chairs" from University of Toulouse ("TEASAO" project). We acknowledge discussions with Boris Dewitte, Sophie Cravatte, Frédéric Marin, Alexis Chaigneau and Peter Haynes. Peter Haynes' visit in Toulouse is funded by the "TEASAO" project.

\section{Appendix A. Derivation of the 1D model}

Different models, with increasing complexities, can be used to study ENSO (see Anderson and McCreary, 1985; Benestad, 1997; Neelin et al., 1998; Dijkstra and Burgers, 2002). In this hierarchy, the simplest ocean model is a 1-layer reduced gravity model, whose equations can be written:

$$
\begin{aligned}
\partial_{t} U+U . \partial_{x} U+V . \partial_{y} U-f V & =\frac{1}{\rho_{r e f}} \partial_{x}\left(g\left(\rho_{s}^{t o t}-\rho_{b}\right) H\right)+F_{x}^{U}, \\
\partial_{t} V+U . \partial_{x} V+V . \partial_{y} V+f U & =\frac{1}{\rho_{r e f}} \partial_{y}\left(g\left(\rho_{s}^{t o t}-\rho_{b}\right) H\right)+F_{y}^{U}, \\
\partial_{t} H+\partial_{x}(H U)+\partial_{y}(H V) & =F^{H}, \\
\partial_{t} \rho_{s}+U \partial_{x} \rho_{s}+V \partial_{y} \rho_{s} & =F^{\rho},
\end{aligned}
$$

where $\vec{U}=(U, V)$ is the horizontal velocity field, $\rho_{\text {ref }}$ is a constant reference density such that the total density in the mixed layer is $\rho_{s}^{t o t}=\rho_{\text {ref }}+\rho_{s}$. The other terms, $\vec{F}^{U}, F^{H}$ and $F^{\rho}$, are terms to take into account all effects necessary to explain the evolution of averaged physical quantities in the upper layer using Eq. A.1, in particular it includes all forcing effects (wind stress, vertical mixing, buoyancy/heat flux, effect of instabilities, ...) and parameterizations. If we consider that the lower layer density variation $\rho_{b}$ is negligible, we can choose $\rho_{\text {ref }}=\rho_{b}$ and Eq. A.1 can be rewritten in the classical form (see for instance Neelin et al., 1998; Dijkstra and Burgers, 


$$
\begin{gathered}
\partial_{t} U+U . \partial_{x} U+V . \partial_{y} U-f V=\partial_{x}(\Theta)+F_{x}^{U}, \\
\partial_{t} V+U . \partial_{x} V+V . \partial_{y} V+f U=\partial_{y}(\Theta)+F_{y}^{U}, \\
\partial_{t} H+\partial_{x}(H U)+\partial_{y}(H V)=F^{H}, \\
\partial_{t} \Theta+\partial_{x}(\Theta U)+\partial_{y}(\Theta V)=F^{\Theta},
\end{gathered}
$$

775

where

$$
\Theta=g\left(\rho_{s}^{t o t}-\rho_{b}\right) H / \rho_{\text {ref }} \simeq g \rho_{s} H / \rho_{\text {ref }}
$$

measures the local buoyancy $(\simeq$ heat) content of the upper layer.

In the previous Eq. A.1 or A.2, we hypothesize that -at first order- the upper ocean mixed layer can be represented as a single vertically homogeneous layer (see Fig. A.15) where physical fields only vary horizontally.

Other hypothesis including more complex averaging of the physical fields in the layer are possible, but even though very simplified, the model and Eq. A.2 take into account the continuity equation and the conservation of momentum and buoyancy (or heat). Horizontal advection and the stretching effect associated with the vertical motion of the base of the thermocline $\left(w(z=-H)=\frac{d H}{d t}\right)$ are taken into account for the evolution of the upper layer thickness and buoyancy content. These are major physical effects for the dynamics of an equatorial Kelvin wave and its impact on the evolution of the density anomaly. Other effects, such as retroactive processes (Ekman feedback or modification of mixing at the base of the thermocline associated with the passage of the wave) are known to be important processes too (Chen et al., 2016). They are associated with the forcing terms $F$ and are not directly represented in the present simplified model but are parameterized as Newtonian cooling terms (see below). 


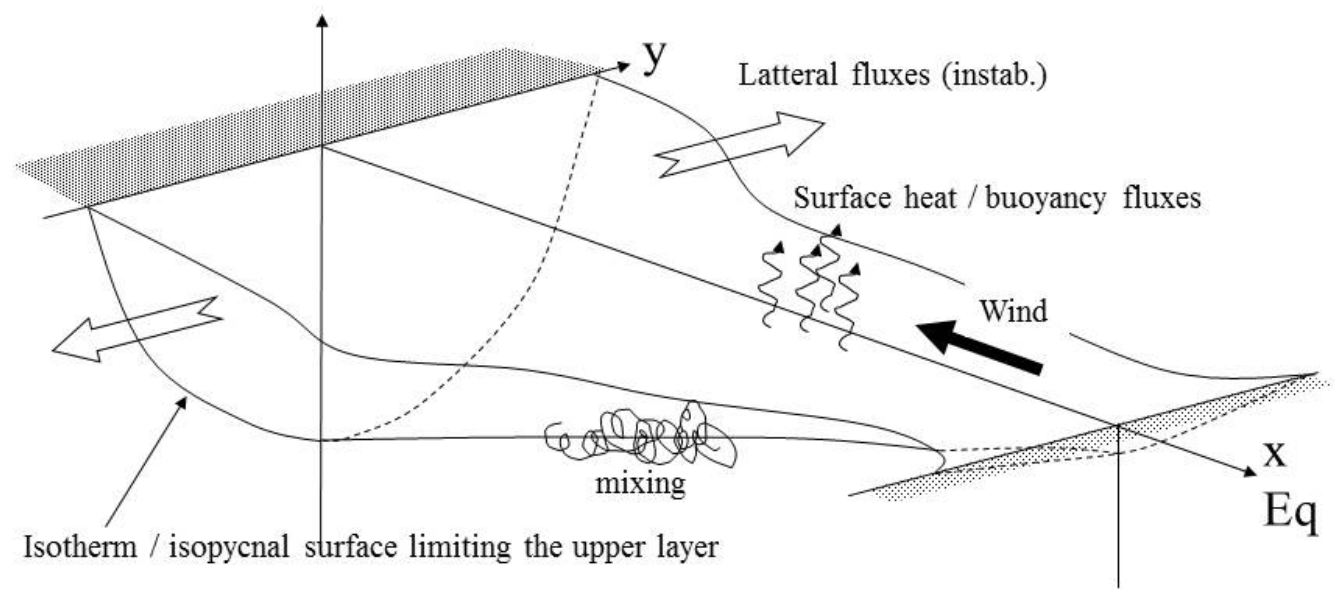

Figure A.15: General view of the mixed layer configuration. 
To concentrate on equatorial Kelvin wave dynamics in variable environ-

which are the final equations we retain for our study.

$$
\begin{aligned}
\partial_{t} U+U . \partial_{x} U & =\partial_{x} \Theta+F^{U}, \\
\partial_{t} H+\partial_{x}(H U) & =F^{H}, \\
\partial_{t}(\Theta)+\partial_{x}(\Theta U) & =F^{\Theta},
\end{aligned}
$$

\section{Appendix B. Equatorial Kelvin Wave solutions for a variable back- ground}

We have been unable to find exact and general solution for the propagation of wave in an environment where the equilibrium state varies spatially and with Newtonian cooling terms. We however propose here an approximate solution which remains quite accurate and allows to estimate the evolution of the wave amplitude. We linearize Eq. 5 and hypothesize that $U_{o}=0$. The equations simply become:

$$
\begin{aligned}
\partial_{t} u & =\partial_{x} \theta-\alpha^{U} u, \\
\partial_{t} \theta & =-\partial_{x}\left(\Theta_{o} u\right)-\alpha^{\Theta} \theta .
\end{aligned}
$$

We then seek for solutions of the form:

$$
\begin{aligned}
& \theta=\theta_{o} e^{-\beta t} e^{i \omega(X(x)-t)}, \\
& u=U(x) \theta .
\end{aligned}
$$


Replacing in Eq. B.1 above yields:

$$
\begin{aligned}
X^{\prime} & =\frac{i \omega+\beta-\alpha^{U}}{i \omega} U, \\
\beta+i \omega & =\alpha^{\Theta}+\left(\Theta_{o} U\right)^{\prime}+\Theta_{o} U^{2}\left(i \omega+\beta-\alpha^{U}\right) .
\end{aligned}
$$

811 We then set $Y=\Theta_{o} U, \beta=\left(\alpha^{\Theta}+\alpha^{U}\right) / 2$ and $\gamma=\left(\alpha^{\Theta}-\alpha^{U}\right) / 2$. This yields:

$$
Y^{\prime}+\frac{i \omega+\gamma}{\Theta_{o}} Y^{2}+i \omega-\gamma=0 .
$$

812 We then hypothesize that $Y=\sqrt{\Theta_{o}}+\delta Y$ with $\delta Y \ll 1 / \sqrt{\Theta_{o}}$ and that $813 \delta Y^{\prime}$ can be neglected when rederiving the equation for $\delta Y$. This yields the 814 approximate solution

$$
\delta Y=-\frac{\Theta_{o}^{\prime}}{4(i \omega+\gamma)}-\frac{\gamma \sqrt{\Theta_{o}}}{i \omega+\gamma},
$$

815 So that at first order

$$
X^{\prime} \simeq \frac{1}{\sqrt{\Theta_{o}}}-\frac{\Theta_{o}^{\prime}}{4 i \omega \Theta_{o}}
$$

816 or

$$
\begin{array}{r}
X \simeq \int \frac{d x}{\sqrt{\Theta_{o}}}-\frac{1}{i \omega} \log \left(\Theta_{o}^{-1 / 4}\right), \\
U \simeq \frac{1}{\sqrt{\Theta_{o}}} .
\end{array}
$$

817 The approximate solutions for $u$ and $\theta$ are then

$$
\begin{aligned}
& \theta=\theta_{o}(t=0) e^{-\beta t}\left[\frac{\Theta_{o}(x=0)}{\Theta_{o}(x)}\right]^{1 / 4} \exp [i \omega(x / C-t)], \\
& u=\frac{\theta}{\sqrt{\Theta_{o}(x)}}=\theta_{o}(t=0) e^{-\beta t} \frac{\Theta_{o}(x=0)^{1 / 4}}{\Theta_{o}(x)^{3 / 4}} \exp [i \omega(x / C-t)](\mathrm{B} .8)
\end{aligned}
$$

where

$$
x / C=\int d x / \sqrt{\Theta_{o}} .
$$


The solution for the layer depth anomaly $h$ can be derived similarly from Eq. 5 and B.8, we finally get the following approximate solutions for the main propagating signal

$$
\begin{aligned}
& \theta=\theta_{o}(x / C(x)-t) e^{-\left(\alpha^{\Theta}+\alpha^{U}\right) t / 2} \frac{C_{o}\left(x=x_{o}\right)^{1 / 2}}{C_{o}(x)^{1 / 2}} \\
& u=\frac{\theta}{C_{o}(x)}=\theta_{o}(x / C(x)-t) e^{-\left(\alpha^{\Theta}+\alpha^{U}\right) t / 2} \frac{C_{o}\left(x=x_{o}\right)^{1 / 2}}{C_{o}(x)^{3 / 2}}, \\
& h=\frac{\rho_{r e f} \theta}{g \rho_{o}(x)}=\theta_{o}(x / C(x)-t) e^{-\left(\alpha^{\Theta}+\alpha^{U}\right) t / 2} \frac{\rho_{r e f} C_{o}\left(x=x_{o}\right)^{1 / 2}}{g \rho_{o}(x) C_{o}(x)^{1 / 2}},(\mathrm{~B} .9)
\end{aligned}
$$

where $\theta_{o}$ is determined by the -fixed- shape of the initial perturbation, typically a gaussian function whose maximum $\theta_{o}^{\max }$ is given, and $x_{o}$ is the initial position of this maximum.

The main perturbation propagates at a speed $C_{o}$ so that at time $t$ the maximum amplitude is positioned at $\Delta x=C_{o} t$. The maximum of the main perturbations as a function of the wave position is thus given by

$$
\begin{aligned}
\theta & \propto \frac{1}{C_{o}^{1 / 2}} e^{-\left(\alpha^{\Theta}+\alpha^{U}\right) x / 2 C_{o}}, \\
u & \propto \frac{1}{C_{o}^{3 / 2}} e^{-\left(\alpha^{\Theta}+\alpha^{U}\right) x / 2 C_{o}}, \\
h & \propto \frac{1}{\rho_{o} C_{o}^{1 / 2}} e^{-\left(\alpha^{\Theta}+\alpha^{U}\right) x / 2 C_{o}} .
\end{aligned}
$$

\section{Appendix C. Density anomaly evolution}

The density anomaly is associated with advection and diabatics effects generated during the passage of the wave. To calculate the density anomaly $\rho$ associated with a linear wave in a variable environment, we thus have to use the equation

$$
\partial_{t} \rho=-u \partial_{x}\left(\rho+\rho_{o}\right)+\frac{\rho_{r e f}}{g\left(h+H_{o}\right)^{2}}\left[\alpha^{H} \Theta_{o} h-\alpha^{\Theta} H_{o} \theta+\left(\alpha^{H}-\alpha^{\Theta}\right) \theta h\right](. C .1)
$$


Linearization of the previous equation yields

$$
\partial_{t} \rho=-u \partial_{x} \rho_{o}+\frac{\rho_{r e f}\left(\alpha^{H}-\alpha^{\Theta}\right)}{g H_{o}} \theta .
$$

833 where $\theta$ and $u=\theta / C_{o}$ are given by Eq. B.9. Thus, starting with a density 834 perturbation which is zero we obtain

$$
\partial_{t} \rho=\left[-\frac{\rho_{o}^{\prime}}{C_{o}^{3 / 2}}+\frac{\rho_{r e f}\left(\alpha^{H}-\alpha^{\Theta}\right)}{g H_{o} C_{o}^{1 / 2}}\right] e^{-\left(\alpha^{\Theta}+\alpha^{U}\right) t / 2} \theta_{o}(x / C(x)-t),
$$

835 which yields using $X=x / C(x)=\int d x / C_{o}$,

$$
\partial_{t} \rho=A(x) \quad G(X-t)
$$

836 where

$$
\begin{aligned}
A(x) & =\left[-\frac{\rho_{o}^{\prime}}{C_{o}^{3 / 2}}+\frac{\rho_{r e f}\left(\alpha^{H}-\alpha^{\Theta}\right)}{g H_{o} C_{o}^{1 / 2}}\right] e^{-\left(\alpha^{\Theta}+\alpha^{U}\right) X / 2} \\
G(X-t) & =e^{\left(\alpha^{\Theta}+\alpha^{U}\right)(X-t) / 2} \theta_{o}(x / C(x)-t)
\end{aligned}
$$

837 The solution of Eq. C.4 is

$$
\rho(x, t)=A(x) \int_{0}^{t} G\left(X-t^{\prime}\right) d t^{\prime} .
$$

838 or using $T=X-t^{\prime}$,

$$
\rho(x, t)=A(x) \int_{X-t}^{X} G(T) d T .
$$

839 Given that the function $\mathrm{G}$ is fixed and localized, the integral term is 840 only important to determine the evolution of the density fields in an area 841 where the propagating gravity wave arrives, but once this perturbation has ${ }_{842}$ passed, the term remains constant. In the area where the wave has passed, 843 the shape of the density anomaly, which generally also correspond to the 844 maximum anomaly reached during the evolution, is then given by

$$
\rho_{\max } \propto\left[-\frac{\rho_{o}^{\prime}}{C_{o}^{3 / 2}}+\frac{\rho_{r e f}\left(\alpha^{H}-\alpha^{\Theta}\right)}{g H_{o} C_{o}^{1 / 2}}\right] e^{-\left(\alpha^{\Theta}+\alpha^{U}\right) X / 2} .
$$


As some forcing fields may only depend on the density anomaly ( $\rho$, and not on the heat content $\theta$ ) it is important to generalize Eq. C.2 and add a Newtonian cooling term depending on the density anomaly alone, to reach:

$$
\partial_{t} \rho=-u \partial_{x} \rho_{o}+\frac{\rho_{r e f}\left(\alpha^{H}-\alpha^{\Theta}\right)}{g H_{o}} \theta+\alpha^{\rho} \rho .
$$

The right hand side term is the forcing term and is not modified. If we set $\tilde{\rho}=\rho e^{-\alpha^{\rho} t}, \tilde{\rho}$ verifies Eq. C.2 and we finally get the following (approximate) solution for $\rho$

$$
\rho_{\max }=\left[-\frac{\rho_{o}^{\prime}}{C_{o}^{3 / 2}}+\frac{\rho_{r e f}\left(\alpha^{H}-\alpha^{\Theta}\right)}{g H_{o} C_{o}^{1 / 2}}\right] e^{-\left(\alpha^{\Theta}+\alpha^{U}\right) X / 2} \quad e^{\alpha^{\rho}(X-t)} \quad \int_{0}^{t} e^{-\alpha^{\rho}\left(X-t^{\prime}\right)} G\left(X-t^{\prime} \backslash \text { (बt' } t^{\prime} 10\right)
$$

1 The same argument as above can be used to state that, since we seek for the maximum density anomaly reached during the passage of the wave, the integral term is constant (only depends on the initial shape of the perturbation). To evaluate the influence of the additional $e^{\alpha^{\rho}(X-t)}$ term, we have to consider three phases at a fixed location (eulerian view):

1. as long as the wave does not attain the location, there is no initial perturbation and the damping term does not act. $\rho$ remains null;

2. when the wave reaches the location, $t \simeq X$ and the density anomaly increases because of advection and previous diabatic terms, but the additional damping term acts during the time of the wave passage $\Delta t=l_{p} / C_{o}$. Thus, in comparison with the case where $\alpha^{\rho}=0$, the maximum density anomaly has to be corrected by a factor $e^{-\alpha^{\rho} \Delta t}=$ $e^{-\alpha^{\rho} l_{p} / C_{o}}$ so that the maximum density anomaly structure reached just after the passage of the wave is given by

$$
\rho_{\max } \propto\left[-\frac{\rho_{o}^{\prime}}{C_{o}^{3 / 2}}+\frac{\rho_{r e f}\left(\alpha^{H}-\alpha^{\Theta}\right)}{g H_{o} C_{o}^{1 / 2}}\right] e^{-\left(\alpha^{\Theta}+\alpha^{U}\right) X / 2} e^{-\alpha^{\rho} l_{p} / C_{o}} .
$$


872 Tables for all model parameters. wave has left the location. with numerical results.

\section{Appendix D. Model Parameters}

3. after the passage of the wave, the forcing term is null and the density anomaly only evolves because of the damping term, so that $\rho=$ $\rho_{\max } e^{-\alpha^{\rho}\left(t-t_{\max }\right)}$, where $t_{\max } \simeq X+l_{p} / C_{o}$ is the time at which the

Equation C.11 is the general analytical formula we use for comparison 


\section{References}

An, S.-I., Jin, F.-F., Aug. 2001. Collective role of thermocline and zonal advective feedbacks in the ENSO mode. J. Clim. 14 (16), 3421-3432.

Anderson, D., McCreary, J., May 1985. Slowly Propagating Disturbances in a Coupled Ocean Atmosphere Model. J. Atm. Sci. 42 (6), 615-629.

Ashok, K., Behera, S. K., Rao, S. A., Weng, H., Yamagata, T., 2007. El nino modoki and its possible teleconnection. J. Geophys. Res. 112, 1-27.

Ashok, K., Yamagata, T., 2009. The el nino with a difference. Nature 461, $481-484$.

Barnier, B., 1998. Forcing the oceans. Proceeding of the NATO advanced study institute on ocean modelling and parameterization, Kluwer academic publishers, $45-80$.

Bellenger, H., Guilyardi, E., Leloup, J., Lengaigne, M., Vialard, J., 2014. ENSO representation in climate models: from CMIP3 to CMIP5. Clim. Dyn. 42 (7-8), 1999-2018.

Benestad, R. E., 1997. Intraseasonal Kelvin Waves in the Tropical Pacific. PhD manuscript, St. Anne's College, Oxford, United Kingdom, 244 pp.

Bosc, C., Delcroix, T., 2008. Observed equatorial rossby waves and ENSOrelated warm water volume changes in the equatorial pacific ocean. Journal of Geophysical Research: Oceans 113 (C6), 1-14. URL http://onlinelibrary.wiley.com/doi/10.1029/2007JC004613/abstract

Busalacchi, A. J., Cane, M. A., 1988. The effect of varying stratification 
895 on low-frequency equatorial motions. Journal of Physical Oceanography $18(6), 801-812$.

Cai, W., Borlace, S., Lengaigne, M., van Rensch, P., Collins, M., Vecchi, G., Timmermann, A., Santoso, A., McPhaden, M., Wu, L., England, M., Wang, G., Guilyardi, E., Jin, F.-F., 2014. Increasing frequency of extreme El Nino events due to greenhouse warming. Nat.Clim.Change 4, 111-116.

Cai, W., Santoso, A., Wang, G., Yeh, S.-W., An, S.-I., Cobb, K., Collins, M., Guilyardi, E., Jin, F.-F., Kug, J.-S., Lengaigne, M., McPhaden, M., Takahashi, K., Timmermann, A., Vecchi, G., Watanabe, M., Wu, L., 2015. Enso and greenhouse warming. Nat. Clim. Change, 849-859.

Capotondi, A., Wittenberg, A., Newman, M., orenzo, E. D. L., Yu, J.-Y., Braconnot, P., Cole, J., Dewitte, B., Giese, B., Guilyardi, E., Jin, F.-F., Karnauskas, K., Kirtman, B., Lee, T., Schneider, N., Xue, Y., Yeh, S.-W., 2015. Understanding ENSO diversity. Bull. Amer. Meteor. Soc., 921-938.

Chen, D., Lian, T., Fu, C., Cane, M., Tang, Y., Murtugudde, R., Song, X., Wu, Q., Zhou, L., May 2015. Strong influence of westerly wind bursts on El Nino diversity. Nature GeoScience 8, 339-345.

Chen, L., Li, T., Behera, S. K., Doi, T., 2016. Distinctive precursory airsea signals between regular and super el niños. Advances in Atmospheric Sciences 33 (8), 996-1004.

Chen, L., Li, T., Wang, B., Wang, L., 2017a. Formation Mechanism for 2015/16 Super El Niño. Nature Scientific Reports 7, 2975.

7 Chen, N., Majda, A., Thual, S., 2017b. Observations and Mechanisms 
of a Simple Stochastic Dynamical Model Capturing El Nino Diversity. J.ClimateAccepted.

Choi, J., An, S.-I., Kug, J.-S., Yeh, S.-W., 2011. The role of mean state on changes in el niño's flavor. Climate Dynamics 37 (5), 1205-1215.

Clarke, A. J., Jan. 2008. An Introduction to the Dynamics of El Nino \& the Southern Oscillation. Academic Press.

Clarke, A. J., Van Gorder, S., Colantuono, G., Apr. 2007. Wind stress curl and ENSO Discharge/Recharge in the equatorial pacific. Journal of Physical Oceanography 37 (4), 1077-1091.

URL http://adsabs . harvard.edu/abs/2007JPO . . . 37.1077C

Cravatte, S., Picaut, J., Eldin, G., 2003. Second and first baroclinic kelvin modes in the equatorial pacific at intraseasonal timescales. Journal of Geophysical Research: Oceans 108 (C8), n/a-n/a, 3266.

URL http://dx.doi.org/10.1029/2002JC001511

Dewitte, B., Choi, J., An, S.-I., Thual, S., Jun 2012. Vertical structure variability and equatorial waves during central Pacific and eastern Pacific El Niños in a coupled general circulation mode. Climate Dynamics 38 (11), $2275-2289$.

URL https://doi.org/10.1007/s00382-011-1215-x

Dewitte, B., Yeh, S.-W., Thual, S., May 2013. Reinterpreting the thermocline feedback in the western-central equatorial Pacific and its relationship with the ENSO modulation. Clim.Dyn. 41 (3-4), 819-830.

Dijkstra, H. A., Burgers, G., 2002. Fluid dynamics of el niño variability. Annual Review of Fluid Mechanics 34 (1), 531-558. 
Djikstra, H., 2006. The ENSO phenomenon: theory and mechanisms. Adv.Geosciences. 6, 3-15.

England, M., McGregor, S., Spence, P., Meehl, G., Timmermann, A., Cai, W., Gupta, A., McPhaden, M., Purich, A., Santoso, A., 2014. Recent intensification of wind-driven circulation in the pacific and the ongoing warming hiatus. Nature Climate Change 4, 222-227.

Fedorov, A., Melville, W., 2000. Kelvin Fronts on the Equatorial Thermocline. J.Phys.Oceano. 30, 1692-1705.

Fedorov, A. V., Hu, S., Lengaigne, M., Guilyardi, E., 2015. The impact of westerly wind bursts and ocean initial state on the development, and diversity of el niño events. Climate Dynamics 44 (5), 1381-1401.

Giese, B., Harrison, D., 1990. Aspects of the kelvin wave response to episodic wind forcing. Journal of Geophysical Research 95, 7289-7312.

Giese, B. S., Sulagna, R., 2011. El nio variability in simple ocean data assimilation (soda), 18712008. Journal of Geophysical Research: Oceans $116(\mathrm{C} 2)$.

Gill, A., 1982. Atmosphere-Ocean Dynamics. San-Diego, USA: Academic Press.

Guilyardi, E., Wittenberg, A., Fedorov, A., Collins, M., Wang, C., Capotondi, A., van Oldenborgh, G. J., Stockdale, T., 2009. Understanding el nio in ocean atmosphere general circulation models: Progress and challenges. Bull. Amer. Meteor. Soc. 90, 325340.

Hirst, A., 1986. Unstable and damped equatorial modes in simple coupled ocean-atmosphere models. J. Atmos. Sci. 43, 606-632. 
Jin, F.-F., Apr. 1997. An equatorial ocean recharge paradigm for ENSO. part i: Conceptual model. Journal of the Atmospheric Sciences 54 (7), $811-829$.

Karnauskas, K. B., Seager, R., Kaplan, A., Kushnir, Y., Cane, M. A., 2009. Observed strengthening of the zonal sea surface temperature gradient across the equatorial pacific ocean. Journal of Climate 22 (16), 4316-4321.

Kug, J.-S., Choi, J., An, S.-I., Jin, F.-F., Wittenberg, A. T., 2010. Warm Pool and Cold Tongue El Nio Events as Simulated by the GFDL 2.1 Coupled GCM. Journal of Climate 23 (5), 1226-1239.

Kug, J.-S., Jin, F.-F., An, S.-I., 2009. Two types of el nio events: Cold tongue el nio and warm pool el nio. Journal of Climate 22 (6), 1499-1515.

Liu, Y., Cobb, K., Song, H., Li, Q., Li, C.-Y., Nakatsuka, T., An, Z., Zhou, W., Cai, Q., Li, J., Leavitt, S., Sun, C., Mei, R., Shen, C.-C., Chan, M.H., Sun, J., Yan, L., Lei, Y., Ma, Y., Li, X., Chen, D., Linderholm, H., 2017. Recent enhancement of central Pacific El Niño variability relative to last eight centuries. Nature Communications 8, 15386.

Long, B., Chang., P., 1990. Propagation of an Equatorial Kelvin Wave in a Varying Thermocline*. J.Phys.Oceano. 20, 1826-1841.

Menkes, C. E., Lengaigne, M., Vialard, J., Puy, M., Marchesiello, P., Cravatte, S., Cambon, G., 2014. About the role of westerly wind events in the possible development of an el nio in 2014. Geophys. Res. Let. 41, 6476-6483.

Neelin, J. D., Battisti, D. S., Hirst, A. C., Jin, F.-F., Wakata, Y., Yamagata, 
T., Zebiak, S. E., 1998. ENSO theory. Journal of Geophysical Research: Oceans 103 (C7), 14261-14290.

Paek, H., Yu, J.-Y., Qian, C., 2016. Why were the 2015/2016 and 1997/1998 extreme El Ninos different. Geophys.Res.Lett. 44, 1848-1856.

Picaut, J., Masia, F., du Penhoat, Y., 1997. An advective-reflective conceptual model for the oscillatory nature of the enso. Sciences 277, 663666 .

Pontaud, M., Thual, O., 1998. Coupled process for equatorial Pacific interannual variability. Q. J. R. Meteorol. Soc. 124, 527-555.

Ren, H.-L., Jin, F.-F., 2013. Recharge oscillator mechanisms in two types of enso. Journal of Climate 26 (17), 6506-6523.

Sohn, B. J., Yeh, S.-W., Schmetz, J., Song, H.-J., Apr 2013. Observational evidences of walker circulation change over the last 30 years contrasting with gcm results. Climate Dynamics 40 (7), 1721-1732.

Su, J., Zhang, R., Rong, X., Min, Q., Zhu, C., 2018. Sea surface temperature in the subtropical pacific boosted the 2015 el niño and hindered the 2016 la niña. Journal of Climate 31 (2), 877-893.

Suarez, M. J., Schopf, P. S., 1988. A delayed action oscillator for ENSO. Journal of the Atmospheric Sciences 45 (21), 3283-3287.

Takahashi, K., Montecinos, A., Goubanova, K., Dewitte, B., 2011. Enso regimes: Reinterpreting the canonical and modoki el nio. Geophysical Research Letters 38 (10), n/a-n/a, 110704.

URL http://dx.doi.org/10.1029/2011GL047364 
Thual, S., Majda, A., Chen, N., Stechmann, S., 2016. A simple stochastic model for el nino with westerly wind bursts. Proc. Nat. Acad. Sci. USA 113, 10245-10250.

Thual, S., Thual, O., Dewitte, B., 2012. Absolute or convective instability in the equatorial pacific and implications for ENSO. Quarterly Journal of the Royal Meteorological Society, n/a-n/a.

Timmerman, A., Oberhuber, J., Bacher, A., Esch, M., Latif, M., Roeckner, E., 1999. Increased el niño frequency in a climate model forced by future greenhouse warming. Nature 398, 694-696.

Wakata, Y., Sarachik, E., 1991. Unstable Coupled Atmosphere-Ocean Basin Modes in the Presence of a Spatially Varying Basic State. J. Atm. Sci. 48, 2060-2077.

Wang, C., Picaut, J., 2004. Understanding enso physicsa review. Earths Climate: The Ocean Atmosphere Interaction, C. Wang, S.-P. Xie, and J. A. Carton, Eds., Amer. Geophys. Union, 2148.

Wu, D.-H., Anderson, D., 1995. Equatorially trapped basin modes on zonally varying thermoclines. Dyn.Atm.Oceans. 21, 279-294.

Xiang, B., Wang, B., Li, T., 2013. A new paradigm for the predominance of standing central pacific warming after the late 1990s. Climate Dynamics $41(2), 327-340$.

Yang, J., O'Brien, J. J., 1993. A coupled atmosphere-ocean model in the tropics with different thermocline profiles. Journal of Climate 6 (6), 10271040. 
Yang, J., Yu, L., 1992. Propagation of Equatorially Trapped Waves on a Sloping Thermocline. J.Phys.Oceano. 22, 573-582.

Yeh, S.-W., Kug, J.-S., Dewitte, B., Kwon, M.-H., Kirtman, B. P., Jin, F.-F., 2009. El Nino in a changing climate. Nature 461, 511-514.

Zebiak, S., Cane, M., May 1987. A Model El Nino-Southern Oscillation. Month. Weath. Rev. 115, 2262-2278.

Zheng, Q., Susanto, R., Yan, X.-H., Liu, W., Ho, C.-R., 1998. Observation of equatorial Kelvin solitary waves in a slowly varying thermocline. N.P.Geophys. 5, 153-165. 


\begin{tabular}{|c|c|}
\hline Variable & Comments \\
\hline \multicolumn{2}{|l|}{$x$ : zonal axis } \\
\hline$y$ : meridional axis & solutions taken at $y=0$ \\
\hline \multicolumn{2}{|l|}{$t$ : time } \\
\hline$U=U_{o}+u:$ zonal current & $u:$ anomaly due to the wave \\
\hline$V:$ meridional current & neglected here \\
\hline$H=H_{o}+h:$ mixed layer depth & $h:$ anomaly due to the wave \\
\hline$P:$ pressure & $P \approx g\left(\rho_{b}-\rho_{s}\right) H$ \\
\hline$\Theta=\Theta_{o}+\theta:$ buoyancy & $\Theta=g\left(\rho_{b}-\rho_{s}\right) H / \rho_{\text {ref }}, \theta:$ anomaly due to the wave \\
\hline$\rho_{s}^{t o t}:$ surface density & $\rho_{s}^{t o t}=\rho_{\text {ref }}+\rho_{s}$ \\
\hline$\rho_{s}=\rho_{o}+\rho:$ surface density variations & $\rho$ is generated by the wave \\
\hline$\rho_{b}$ : lower layer density & we choose $\rho_{r e f}=\rho_{b}$ \\
\hline$F_{x}^{U}:$ zonal momentum forcing & also noted $F^{U}$ \\
\hline \multicolumn{2}{|l|}{$F_{y}^{U}:$ meridional momentum forcing } \\
\hline \multicolumn{2}{|l|}{$F^{H}$ : mixed layer depth forcing } \\
\hline \multicolumn{2}{|l|}{$F^{\Theta}$ : buoyancy forcing } \\
\hline \multicolumn{2}{|l|}{$F^{\rho}:$ density forcing } \\
\hline$\delta F$ : external forcing anomalies & $F^{U}=F_{o}^{U}(x)+\delta F^{U}(x, t)$, etc \\
\hline
\end{tabular}

Table D.2: Model variables definitions. 


\begin{tabular}{|c|c|}
\hline Parameter & Comments \\
\hline$g$ : gravity & $g=9.81 m . s^{-2}$ \\
\hline$f:$ Coriolis force & $f=0$ at the equator \\
\hline$U_{o}$ : background zonal current & $U_{o}=0$ in analytical calculations \\
\hline$L:$ basin length & $L=30.000 \mathrm{~km}$ \\
\hline \multicolumn{2}{|l|}{$x_{o}:$ initial position of wave } \\
\hline$H_{o}:$ background depth & $\rho_{o}=H_{\text {mean }}+\Delta H_{\max } \frac{x-L / 2}{L}$ \\
\hline$H_{\text {mean }}:$ mean mixed layer depth & in general $H_{\text {mean }}=120 \mathrm{~m}$ \\
\hline$\Delta H_{\max }:$ linear depth variation & in general $\Delta H_{\max }=160 \mathrm{~m}$ \\
\hline$\rho_{\text {ref }}:$ reference density & $1000 \mathrm{~kg} \cdot \mathrm{m}^{-3}$ \\
\hline$\rho_{o}$ : background density & $\rho_{o}=\rho_{\text {mean }}+\Delta \rho_{\text {max }}^{l i n} \frac{x-L / 2}{L}+\Delta \rho_{\text {max }}^{t h} t h\left(\left(x-x_{t h}\right) / L_{t h}\right)$ \\
\hline$\rho_{\text {mean }}:$ mean mixed layer density & in general $\rho_{\text {mean }}=-3 \mathrm{~kg} / \mathrm{m}^{3}$ \\
\hline$\Delta \rho_{\text {max }}^{\text {lin }}:$ linear density variation & $\Delta \rho_{\max }^{l i n}=3 \mathrm{~kg} / \mathrm{m}^{3}$ or $=0.5 \mathrm{~kg} / \mathrm{m}^{3}$ \\
\hline$\Delta \rho_{\max }^{t h}:$ nonlinear density variation & in general $\Delta \rho_{\max }^{t h}=0 \mathrm{~kg} / \mathrm{m}^{3}$ or $=3 \mathrm{~kg} / \mathrm{m}^{3}$ \\
\hline$L_{t h}:$ front lengthscale & in general $L_{t h}=5000 \mathrm{~km}$ \\
\hline$x_{t h}:$ front position & $x_{t h}=L / 2$ \\
\hline$\rho_{o}^{\prime}, \rho_{o}^{\prime \prime}:$ first and second derivatives & $d_{x} \rho_{o}, d_{x}^{2} \rho_{o}$ \\
\hline$\Theta_{o}$ : background buoyancy & $\Theta_{o}=g \rho_{o} H_{o} / \rho_{r e f}$ \\
\hline$\alpha:$ Newtonian coolings parameters & $\delta F^{U}=-\alpha^{U} u$, etc \\
\hline$C_{o}$ : background phase speed & $C_{o}(x)=\sqrt{-g \rho_{o} H_{o} \rho_{r e f}}$ \\
\hline$C:$ analytical phase speed & $x / C(x)=\int_{x_{o}}^{x} d s / C_{o}(s)$ \\
\hline
\end{tabular}

Table D.3: Model parameters definitions. 